\title{
Materials International Journal of Material Forming
}

\section{Thermo-mechanical characterization of unsaturated polyester/glass fiber composites for recycling}

\author{
A. Nouigues ${ }^{1,2}$, E. Le Gal La Salle ${ }^{1,2}$, J-L. Bailleul ${ }^{2}$ \\ 1. Icam de Nantes, 35 avenue du champ de Manœuvres, 44470 Carquefou, Nantes \\ 2. LTeN, UMR CNRS 6607, Rue Christian Pauc, 44306, Nantes \\ arbia.nouigues@icam.fr, eric.legallasalle@icam.fr, jean-luc.bailleul@univ-nantes.fr
}

\begin{abstract}
A b s t r act
Unsaturated-polyester/glass-fiber composites are worldwide more and more used. Actually, their thermochemical and mechanical recycling paths are quite well-known. But, due to the relatively low value of the end products, these two paths are hardly economically viable. Thus, a third way will be proposed as a thermomechanical path. In order to recycle these composites, a study of their thermo-mechanical behaviors is proposed in this work, through various static and dynamic tests at temperatures ranging from ambient to $150^{\circ} \mathrm{C}$, over their glass-transition-temperature $\left(\mathrm{T}_{\mathrm{g}}\right)$. As expected, the results prove that unsaturated-polyester reinforced by woven glass-fibers is more resistant in static flexion and traction than composite made with mixed chopped and woven glass-fibers, at ambient temperature and up to $150^{\circ} \mathrm{C}$. Also, static and dynamic tests have shown that composite manufactured by infusion is more resistant than the hand lay-up composite forming. Indeed, dynamic 3-points-flexural-bending test indicates that these composites lose their stiffness during the temperature rise.

Furthermore, the composites ageing study shows the appearance of Mullins effect and hysteresis phenomena, at $150^{\circ} \mathrm{C}$. After an accelerated ageing in distilled water at ambient temperature, the composite lost about $66 \%$ of its flexural resistance but a gain of $195 \%$ in its deformation at break is obtained. For an ageing in distilled water at temperature around its $\mathrm{T}_{\mathrm{g}}$, this composite lost the same ratio in its flexural resistance but got $180 \%$ in its yield deformation, compared to its virgin state. The mechanical properties lose can be interpreted by the ester function hydrolysis and the glass fibers damage.
\end{abstract}

Keywords: Glass fibers/Polyester, Recycling, Thermo-mechanical properties, High Temperatures, Ageing behavior

\section{Introduction}

Composite materials are known to be one of the materials of the future. Their utilization is constantly increasing in the majority of industrial fields such as aeronautics, marine, automotive, transport... The most produced type of composite is the unsaturated polyester glass fibers reinforced composite, compared to carbon fibers reinforced epoxy composites and thermoplastic composites [1]. However, with this important production due to its large use, there is a significant waste stream of polyester glass fibers reinforced composites. This stream of composites wastes is divided into two families: production wastes (new) and end of life wastes (old) [2]. Actually, they face many problems in their recycling. $40 \%$ of these wastes are stored, $25 \%$ undergo incineration and only $35 \%$ are recycled [3]. But even though, the recycling methods are not $100 \%$ satisfying. Currently, the main studied and applied technique to recycle polyester glass fibers reinforced composites are the grinding as a mechanical process, the pyrolysis as a thermal process and the solvolysis as a chemical process [4]. But all these processes have many negative points which make the recycling of these composite materials difficult. For example, the grinding generates the deterioration of the mechanical properties of glass fibers and induces a lot of wear of the tools [2]. It is also an expensive technique since the recuperated product can be a filler or a reinforcement. The use of filler is not commercially reliable because the cost of virgin fillers is very low such as calcium carbonate or silica [4]. In addition, their applications are limited, so that almost the polyester glass fibers reinforced composites wastes are grinded and then used for building as an additive in concrete $[5,6]$. 
For pyrolysis, the resin is transformed into lower-weight molecules at temperatures between over $400^{\circ} \mathrm{C}$, producing mainly many gases such as carbon dioxide, hydrogen and methane, and an oil fraction [4]. The products of pyrolysis coming from the resin can be used as a source of energy. Part of them, usually the gas, is used to run the process. In oxidative conditions, the composite can be used as a fuel and a material source in cement factories. $10 \%$ of the fuel input used to make a cement could be substituted by polymer composites reinforced with glass fibers [4]. Since, the fibers have various sensitivities to processing conditions, depending on their type and the working temperature, the mechanical properties of the glass fibers are decreased by at least $50 \%$, especially as the minimal process temperature is over $300^{\circ} \mathrm{C}$.

The solvolysis [7] is a chemical treatment using a solvent to degrade the resin. The main drawback of this process is the cost of the devices induced by the severity of the conditions. When the conditions are supercritical, for example, water reactors become expensive due to raison that they have to withstand high temperatures (above $374^{\circ} \mathrm{C}$ ) and pressures (above $22.1 \mathrm{MPa}$ ), as well as corrosion. Indeed, the used solvents and the catalysts can be toxic and difficult to dispose or to separate. Moreover, working with some catalysts can damage the fibers whereas the main objective of solvolysis is to recover [4]. However, for all the previous recycling methods, the recycling is not applied to end of life materials, partially due to the collecting cost of the goods, which impairs the overall economic efficiency of the industrial sector.

So today, the challenge of these composite materials is to find an efficient recycling method to minimize their environmental impact, by reducing their waste stock. This recycling technique should avoid the maximum of all the previous recycling processes disadvantages. Thus, the idea is to find a new method to recycle polyester glass fibers reinforced composites. To reduce the industrial operations, a thermo-mechanical recycling technique is looked for. This technique is based on the forming of composite at high temperature like the hot stamping. Two of the co-authors were already involved in an applied research project, which lead to an industrial process [8]. In this process, chunks of composites are hot melted with recycled thermoplastics. Many shadows remain in the behavior of the composite in this process. So, the goal of this article is to bring some information on the characteristics of the polyester glass fibers reinforced composites, helping to understand their behaviors underin different the thermomechanical processes recycling conditions to determine its parameters to adopt it to composite. $\underline{\text { So, a mechanical characterization of composite is required to determine the composite mechanical properties at }}$ high temperatures.

Based on the previous, in this article, we propose a study of this type of new or laboratory aged composite behavior under different conditions to identify its parameters to model any thermomechanical recycling process.

The behavior of unsaturated polyester resin reinforced with glass fibers composite depends on many parameters like volume fraction, type of glass fibers layers (chopped or roving), manufacturing process, temperature... It is shown in literature [9] that the mechanical properties of E-glass (chopped) reinforced polyester composites manufactured by hand-lay-up, were improved while increasing the glass fibers percentages, at ambient temperature. Moreover, the chopped (mat) and the continuous glass fibers showed different behaviors, depending on the applied mechanical test [10]. Besides that, the manufacturing process has also an influence on the composite behavior. M. Davallo and H. Pasdar [11] studied the mechanical properties of glass fibers/polyester composites formed by hand-lay-up and by resin transfer molding. They found that composites manufactured by hand-lay-up are more resistant on flexural test than composites manufactured by transfer molding.

On account of the temperature importance on the composite behavior, Laoubi and al. [12] reported a study on the chemical, mechanical and physical properties of unsaturated polyester reinforced E-glass fibers composites, between $130^{\circ} \mathrm{C}$ and $400^{\circ} \mathrm{C}$. They observed an improvement of the young modulus measured in three point bending test between $130^{\circ} \mathrm{C}$ and $200^{\circ} \mathrm{C}$ and a drop of the properties at $280^{\circ} \mathrm{C}$. It is suggested that the evolutions of the properties are due to post curing below $100^{\circ} \mathrm{C}$, a water evaporation and some cracks in the resin-fibers interphase at $200^{\circ} \mathrm{C}$ and a matrix degradation above. It was shown by Dhakal et al. [13] that the water absorption behavior radically alters the mechanical properties of unsaturated polyester hemp fibers reinforced composite, at $100^{\circ} \mathrm{C}$. It is due to the important degradation of the mechanical properties of unsaturated polyester hemp glass fibers, induced by moisture. So, the moisture generates significant drops in both tensile and flexural properties, due to the degradation of the fiber-matrix interface.

Since there are few works that have examined the mechanical behavior of mixed chopped and continuous glass fibers reinforced polyester, while varying the temperature and the manufacturing process, this research focuses on unsaturated polyester glass fibers reinforced composites behavior as function of the temperature level (between 
$\mathrm{T}_{\text {amb }}$ and $150^{\circ} \mathrm{C}$ ), using two different shapes of glass fibers (chopped and woven roving) and two elaborating processes: hand lay-up (molding) and infusion.

We have chosen to study 3 types of composites: two composites have the same composition but they were elaborated by two different processes, to determine the influence of process on composite behavior. And the other one has a composition adopted in manufacturing of boat hull $[14,15]$ and blades of wind turbines, to analyze an industrial example of composite. The characterization is made mainly by several thermo-mechanical techniques which are: static and cyclic tensile tests, static and dynamic bending, while varying the temperature, and differential scanning calorimetry (DSC). Indeed, a treatment of the influence of the water absorption on the thermomechanical properties of composite, a laboratory process of ageing is carried out in this paper, at different temperatures.

\section{Materials and experimental methods}

\subsection{Materials:}

Composites studied in this work are composed of unsaturated polyester resin (table 1) with volume fraction of $60 \%$ and glass fibers with volume fraction of $40 \%$ in form of woven taffetas $\left(300 \mathrm{~g} . \mathrm{m}^{2}\right)$ and mat $(100,300,450$ g. $\mathrm{m}^{-2}$ ). The mechanical properties of glass fibers are presented at table 2. For the molding, two hand lay-up variants (LU1 and LU2) are studied and for the infusion, only one variant (IN) is used. The compositions of these different variants are presented in Table 4 and are chosen to be representative of the industrial compositions as well as the chosen manufacturing processes. IN and LU2 have the same composition to compare the influence of the process. LU1 and LU2 compositions were chosen to be able to compare the influence of the mat and the waving glass fibers reinforcements on composites behaviors.

The $1^{\text {st }}$ composition, LU1, was chosen to be close to leisure boats applications $[14,15]$. So, a real industrial example of composites thermosetting is studied in this article to try to get some knowledge about what a real behavior in an industrial process would. The used unsaturated polyester resin is an isophthalic, thixotropic and pre-accelerated polyester resin. It presents very good chemical qualities and a good heat resistance with a good resistance to ageing. A formulation of the resin is used for the hand lay-up process and another for the infusion (table 3).

\subsection{Manufacturing processes:}

The used composites in this study are manufactured in the laboratory of composite manufacturing at ICAM Nantes by the hand lay-up (Fig.1.) and the infusion process. In the hand lay-up, a roll stock fibers glass reinforcement is manually placed on the mold. The resin is applied using a paint roller. Subsequent layers of fibers glass reinforcement are added to build laminate thickness. The other mats are placed then on the preceding polymer layer and pressured using a roller to remove any trapped air bubbles and the excess of polymer as well.

After curing at room temperature, the composite is removed from the mold surface.

In the infusion process, the whole dry composite layers are vacuum bagged. Once bag leaks have been eliminated, resin is allowed to flow into the laminate. The resin distribution over the whole laminate is aided by resin flowing easily through the non-structural fabric, and wetting the fabric out from above.

Table 1 : Mechanical properties of unsaturated polyester resin of hand lay-up

\begin{tabular}{|c|c|c|c|c|c|}
\hline Density & $\begin{array}{c}\text { Bending } \\
\text { resistance }\end{array}$ & $\begin{array}{c}\text { Tensile } \\
\text { strength }\end{array}$ & $\begin{array}{c}\text { Elongation } \\
\text { at rupture }\end{array}$ & $\begin{array}{c}\text { Elastic } \\
\text { return }\end{array}$ & $\begin{array}{c}\text { Viscosity at } \\
\mathbf{5} \mathbf{~ r p m}\end{array}$ \\
\hline $1.1 \mathrm{~g} / \mathrm{cm} 3$ & $102 \mathrm{MPa}$ & $80 \mathrm{MPa}$ & $4 \%$ & $7 \%$ & $1750 \mathrm{mPa} . \mathrm{s}$ \\
\hline
\end{tabular}


Table 2 : Mechanical properties of E-glass [13]

\begin{tabular}{|c|c|c|c|}
\hline Density & $\begin{array}{c}\text { Young } \\
\text { modulus }\end{array}$ & Tensile strength & $\begin{array}{c}\text { Elongation at } \\
\text { break }\end{array}$ \\
\hline $2.5 \mathrm{~g} / \mathrm{cm} 3$ & $70 \mathrm{GPa}$ & $2000-3500 \mathrm{MPa}$ & $2.5 \%$ \\
\hline
\end{tabular}

Table 3 : Mechanical properties of unsaturated polyester resin of infusion

\begin{tabular}{|c|c|c|c|c|}
\hline Density & $\begin{array}{c}\text { Bending } \\
\text { resistance }\end{array}$ & $\begin{array}{c}\text { Tensile } \\
\text { strength }\end{array}$ & $\begin{array}{c}\text { compressive } \\
\text { strength }\end{array}$ & Viscosity \\
\hline $1.12 \mathrm{~g} / \mathrm{cm} 3$ & $90 \mathrm{MPa}$ & $70 \mathrm{MPa}$ & $4 \%$ & $\begin{array}{c}300-400 \\
\mathrm{mPa} . \mathrm{s}\end{array}$ \\
\hline
\end{tabular}

Table 4 : Compositions of different manufactured composites

\begin{tabular}{|c|l|l|l|}
\hline Variant & \multicolumn{1}{|c|}{ LU1 } & \multicolumn{1}{c|}{ LU2 } & \multicolumn{1}{c|}{ IN } \\
\hline \multirow{3}{*}{ Composition } & $\begin{array}{l}\text { 1 mat } 100 \mathrm{~g} \cdot \mathrm{m}^{-2} \\
2 \text { mat } 300 \mathrm{~g} \cdot \mathrm{m}^{-2}\end{array}$ & 6 woven 300 & g.m woven \\
& 2 mat $450 \mathrm{~g} \cdot \mathrm{m}^{-2}$ & & $300 \mathrm{~g} \cdot \mathrm{m}^{-2}$ \\
& 1 woven $300 \mathrm{~g} \cdot \mathrm{m}^{-2}$ & & \\
& & & \\
\hline
\end{tabular}

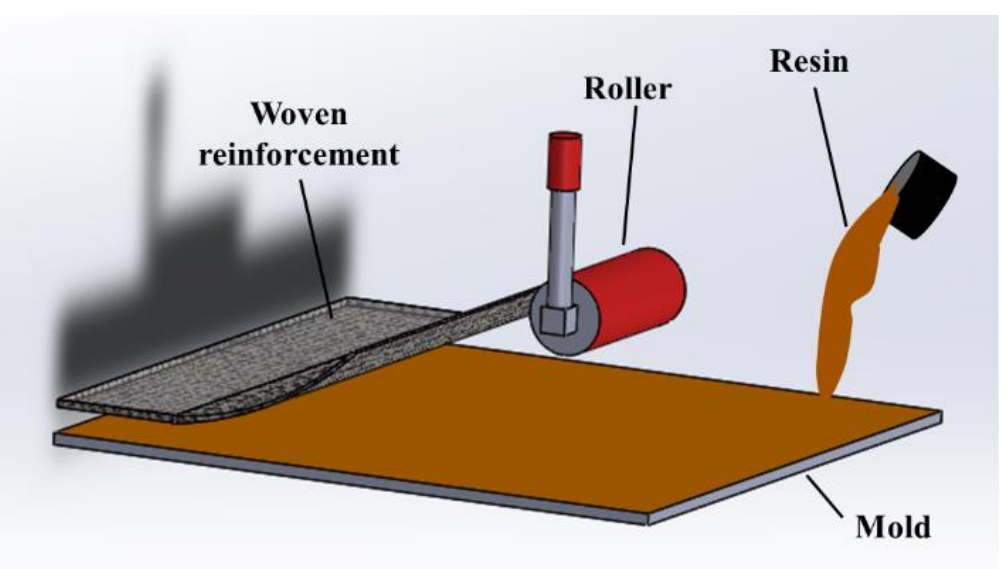

Fig. 1 : Explanatory schema of hand lay-up process

\subsection{Experimental techniques and analysis}

\subsubsection{Thermal study by Differential Scanning Calorimetry (DSC)}

DSC test is a thermal analyze. It is used to understand the morphology of composites by measuring their thermal transitions. Thus, we can determine their glass-transition temperature $(\mathrm{Tg})$, their crystallization temperature $(\mathrm{Tc})$ or melting temperatures (Tm), their specific heat capacities $\mathrm{Cp}$ and their degree of transformation.

Given that the cross linking of unsaturated polyester resin is never complete at ambient temperature $\left(\mathrm{T}_{\mathrm{amb}}\right)$ [16], we have tried several protocols to ensure a nearly complete cross linking. These cycles are shown in Fig.2. For each protocol, 5 samples were used. The ready-to-use resin is put in the DSC oven and the temperatures described in figure 2 are imposed by the oven. For cycles 2 to 5 , the resin is allowed to cure at a given temperature and a given time, then, after cooling at $30^{\circ} \mathrm{C}$, a measuring step is performed by heating up to $200^{\circ} \mathrm{C}$. Cycle 1 measures the signal of an uncured resin, to evaluate the total transition enthalpy $\Delta \mathrm{H}$ of raw resin, using the first cycle protocol Cy1 (Fig.2). After finishing each protocol, the cross linking rate defined as $\alpha$ is calculated as function of transition enthalpy $\Delta H$. 


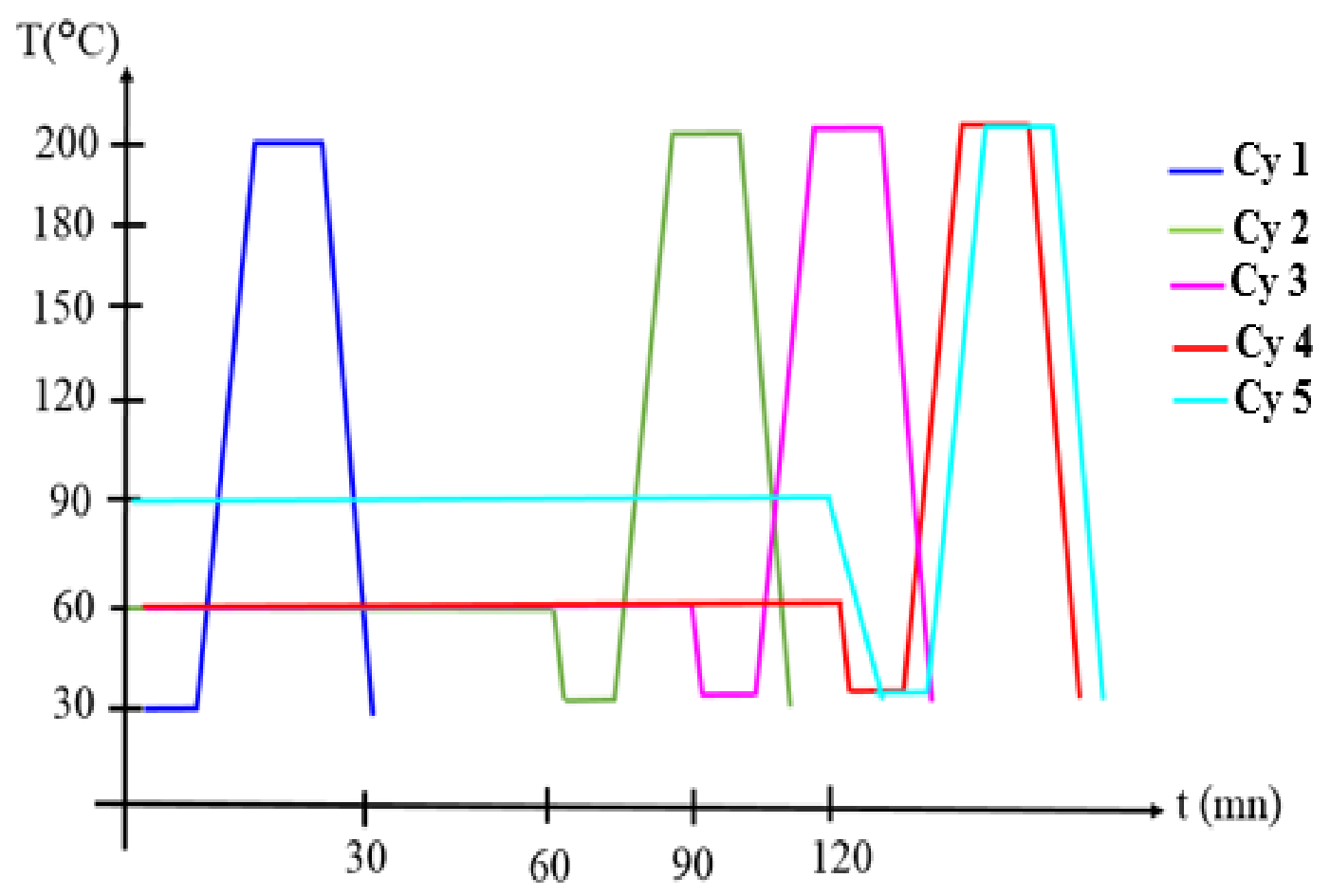

Fig. 2 : DSC temperature cycles

The measuring protocol was applied to the specimens of composite and also to the specimens of polymerized resins:

- Holding the temperature at $30^{\circ} \mathrm{C}$ for $5 \mathrm{~min}$ (step)

- Raising the temperature from $30^{\circ} \mathrm{C}$ to $200^{\circ} \mathrm{C}$ at a heating rate of $2^{\circ} \mathrm{C} / \mathrm{min}$ (ramp)

- Holding the temperature at $200^{\circ} \mathrm{C}$ for $5 \mathrm{~min}$ (step)

- Cooling from $200^{\circ} \mathrm{C}$ to $30^{\circ} \mathrm{C}$ at a cooling rate of $20^{\circ} \mathrm{C} / \mathrm{min}$

The speed of heating is low to guarantee the temperature homogeneity and uniformity inside our composite. The enthalpy of reaction is measured during this heating ramp. Also, for the cooling, the speed is relativity high to prevent any chemical reaction that may happen in case of future measurements on the specimen.

\subsubsection{Thermo-mechanical methods}

\section{Static study}

\section{- Tensile test}

Static tensile tests are done for each composite, until break. The specimens used for tests, have a rectangular shape, according to the NF EN ISO 527- 4 standard and they were tested at different temperatures on an INSTRON® testing machine. For this test, 5 to 10 samples were used.

\section{- 3 point flexural bending test}

The bending tests are realized on an INSTRON testing machine at ambient temperature $\left(\mathrm{T}_{\mathrm{amb}}\right)$ in accordance with the standard NF EN ISO 14125: 1998 (F). The test specimens are also cut according to this standard and have the following dimensions: $(72 \times 15 \times 3.6) \mathrm{mm}^{3}$ for LU1, $(40 \times 15 \times 2) \mathrm{mm}^{3}$ for LU2 and $(72 \times 15 \times 3) \mathrm{mm}^{3}$ for IN. For this flexion test, 5 to 10 samples were used.

\section{$\checkmark$ Dynamic study: Dynamic Mechanical Analysis (DMA)}

DMA is a method of thermo-mechanical analysis that allows the determination of the mechanical properties of materials according to temperature. For analyzing the thermodynamic behavior of our composite materials at 
different temperatures, we have chosen the 3-points bending to load rectangular specimens at constant frequency (5Hz) using a Metravib ® VA2000 machine. For this type of test, 5 to 10 samples were used.

\section{$\checkmark$ Study of ageing behavior}

\section{- Mechanical ageing: Cyclic tensile of variant LU1}

For this study, fatigue tensile tests are done at $T_{\text {amb }}$ on INSTRON testing machine, using a speed of $5 \mathrm{~mm} / \mathrm{min}$. The aim of these tests is to evaluate the behavior of the composites during the first cycles of a cyclic loading mode. The specimens used have a rectangular shape. For this study, 5 to 10 samples were used.

\section{- Thermochemical ageing: hydrolysis of LU1}

The hydrolysis technique is used to study the effect of ageing composites immersed in water. We only used the composite LU1 to get an idea about the ageing behavior of our composite. Three test protocols are performed. The $1^{\text {st }}$ test protocol consists in putting the composite specimens in distilled water at ambient temperature until their total water saturation, $\mathbf{S}_{\mathbf{t}}$. In our case, the total water saturation rate indicates the amount of water presented in composite (water content). To accelerate the ageing of composite and to determine the influence of temperature on its behavior, we have chosen $80^{\circ} \mathrm{C}$ and $90^{\circ} \mathrm{C}$ as working hydrolysis temperatures, because they are close to the glass transition temperature, $\mathrm{T}_{\mathrm{g}}$, of our composite, which is around $100^{\circ} \mathrm{C}$. So, the $2^{\text {nd }}$ test protocol refers to the immersion of the composite specimens in distilled water at $80^{\circ} \mathrm{C}$ until their total water saturation. The $3^{\text {nd }}$ test protocol refers to the immersion of the composite specimens in distilled water at $90^{\circ} \mathrm{C}$ until their total water saturation in mass. The water saturation rate is calculated based on the initial mass of the specimen. Thus, every day, the composite specimens are weighed after wiping. Then, we calculate their water saturation rates which are equal to:

$$
\mathrm{S}_{\mathrm{t}}=\frac{\left(\mathrm{m}_{\mathrm{n}}-\mathrm{m}_{0}\right)}{\mathrm{m}_{0}} \times 100 \text {, with } \mathrm{m}_{\mathrm{n}} \text { is the new sample mass and } \mathrm{m}_{0} \text { is its mass before ageing. }
$$

The $1^{\text {st }}$ test took one month to reach the $\mathbf{S}_{\mathbf{t}}$ of all the specimens. Then, they are left for two months and 20 days at $\mathrm{T}_{\text {amb }}$ to dry.

For the $2^{\text {nd }}$ test, the composite samples remained at $80^{\circ} \mathrm{C}$ for 20 days until the water saturates the samples. Then they were dried at $T_{a m b}$. There are 4 samples which are dried at $T_{\text {amb }}$ for 3 days and 3 samples which are dried for 10 days, to determine the effect of the drying period on the water aged composite behavior.

The samples of the $3^{\text {rd }}$ test were put at $90^{\circ} \mathrm{C}$ for 14 days until their total water saturation. Then, they are $t$ in the oven for 3 hours and 3 minutes at $90^{\circ} \mathrm{C}$ to accelerate their drying.

All the dried samples are tested on the 3 point flexural bending at $T_{\text {amb }}$. Before the test of each samples of each protocol test, we measured their masses and then we calculated their final water saturation rates. Their dimensions are: $75 \times 15 \times 3.8\left(\mathrm{~mm}^{3}\right)$.

\section{Results and discussion:}

\subsection{DSC}

After applying the previous protocols to unsaturated polyester resin, Table 5 shows the rate of resin cross-linking. The total transition enthalpy $\Delta \mathrm{H}_{\text {total }}$ is evaluated from cycle of resin before polymerization by calculation of the area under the exothermic curve. Then, the cross linking rate $\alpha$ is calculated as function of the total transition enthalpy $\Delta \mathrm{H}_{\text {total }}$ and the residual transition enthalpy $\Delta \mathrm{H}_{\text {residual. }}$. It is given that:

$$
\Delta \mathrm{H}_{\text {residual }}=\left(\mid \Delta \mathrm{H}_{\text {before polymerization }}-\Delta \mathrm{H} \text { cycle } \mid\right)
$$

So, the cross linking rate defined as: $\boldsymbol{\alpha}=\left(\mathbf{1}-\left|\frac{\Delta \mathrm{H} \text { residual }}{\Delta \mathrm{H} \text { total }}\right|\right)$ 
Table 5 : DSC unsaturated polyester resin

\begin{tabular}{|c|c|c|c|c|c|}
\hline \multirow{2}{*}{} & \multirow{2}{*}{$\begin{array}{c}\text { Before } \\
\text { polymerization } \\
(\mathbf{C y} \text { 1) }\end{array}$} & \multicolumn{4}{|c|}{ After polymerization at Tamb } \\
\cline { 3 - 6 } & $\mathbf{C y ~ 2}$ & $\mathbf{C y ~ 3}$ & $\mathbf{C y}$ 4 & Cy 5 \\
\hline$|\Delta \mathrm{H}|(\mathbf{J} / \mathbf{g})$ & 25.7 & 6.8 & 5.6 & 5.4 & 1.6 \\
\hline $\boldsymbol{\alpha}$ & & 0.973 & 0.978 & 0.989 & 0.993 \\
\hline
\end{tabular}

We conclude that we can obtain a quasi-cross-linked resin (composite), if we put it at $90^{\circ} \mathrm{C}$ during two hours. The Table 6 summarizes the specific heat capacities of two resins and of different composites between $30^{\circ} \mathrm{C}$ and $150^{\circ} \mathrm{C}$ and their transition temperatures $\left(\mathrm{T}_{\mathrm{g}}\right)$.

Table 6 : DSC of different resins and composites

\begin{tabular}{|c|c|c|c|}
\hline & $\begin{array}{c}\mathbf{C}_{\mathbf{p}} \text { resin } \\
\mathbf{J} /(\mathbf{k g} \cdot \mathbf{K})\end{array}$ & $\begin{array}{c}\mathbf{C}_{\mathbf{p}} \text { composite } \\
\mathbf{J} /(\mathbf{k g} . \mathbf{K})\end{array}$ & $\mathbf{T}_{\mathbf{g}}\left({ }^{\circ} \mathbf{C}\right)$ \\
\hline Hand-Lay-up & $1500^{ \pm 250}$ & $1200^{ \pm 250}$ & 123 \\
\hline Infusion & $1400^{ \pm 200}$ & $1100^{ \pm 200}$ & 127 \\
\hline
\end{tabular}

\subsection{Static study}

\subsubsection{Tensile test}

\section{$\checkmark \quad$ At $_{\text {amb }}(V=5 \mathrm{~mm} / \mathrm{min})$}

Fig. 3 shows the results of tensile test for variant LU2 at $T_{a m b}$. It has a rigid behavior at $T_{a m b}$ and we can mainly distinguish two zones:

Zone (1): it is quasi-linear and it corresponds to the elastic deformation of the material. But there is a curved and non-linear zone. This could be due to a progressive alignment of the microstructures of the material with the traction axis and a reorganization of the amorphous chains [17].

Zone (2): it corresponds to the zone of rupture. The rupture of these materials is brittle.

Fig. 4 shows the average curves over 10 samples of different variants. The average of these curves is the average of stress of all samples of each variant, in each deformation rate. We calculate the error bar in some points, which is equal to maximum stress in that point minus the average.

We notice that the three variants have a rigid behavior at $T_{\text {amb }}$. All the mechanical properties of our variants in tensile at $\mathrm{T}_{\mathrm{amb}}$ are presented at Table 7. However, the LU2 and IN are more resistant in tension than the variant LU1. So, we can conclude that the resistance of woven roving glass is greater than the resistance of mats. For the break, the 3 variants have a sudden break.

\section{$\checkmark \quad \operatorname{At~} 120^{\circ} \mathrm{C}(\mathrm{V}=5 \mathrm{~mm} / \mathrm{min})$}

At $120^{\circ} \mathrm{C}$, the behaviors of all the different variants change from the glassy to the rubbery state with a remarkable drop in maximum tensile stress at fracture (40\%).

The yield strain being almost unchanged, as shown in Fig. 5.

Fig. 8 summarizes the fracture modes of each variant. For LU2 and IN, there is the appearance of delaminating phenomenon of glass layers. The LU1 is characterized by a sliding failure mode. Table 8 shows the mechanical properties of all variants in tensile tests at $120^{\circ} \mathrm{C}$. So, the temperature $120^{\circ} \mathrm{C}$ which is superior to $\mathrm{T}_{\mathrm{g}}$ composite, 
damage its resistance, but it gives more compliance and a remarkable plastic zone.

\section{$\operatorname{At} 150^{\circ} \mathrm{C}(\mathrm{V}=5 \mathrm{~mm} / \mathrm{min})$}

At $150^{\circ} \mathrm{C}$, the behavior of different variants (Fig. 9) remains rubbery but with a maximum-tensile-stress dropping by $30 \%$ and a strain yield increasing by $15 \%$, comparing to the behavior at $120^{\circ} \mathrm{C}$ (Fig. 7).

If we are interested in the fracture mode, we can observe it in Fig. 8 which presents the rupture of 3 variants. For the rupture of IN and of LU2, the phenomenon of delaminating of the reinforcing layers is noted and it is total. For the LU1, the fracture mode change. Table 9 presents all the mechanical properties of our variants in tensile at $150^{\circ} \mathrm{C}$. So, as expected, the elevated temperature changes the behavior of the composites and decreases its mechanical properties. But in other hand, the appearance of a large plastic zone can help in its forming.

Table 7 : Mechanical properties of variants at $\mathrm{T}_{\mathrm{amb}}$

\begin{tabular}{|c|c|c|}
\hline & E (GPa) & $\boldsymbol{\sigma}_{\mathbf{m}}(\mathbf{M P a})$ \\
\hline LU1 & $7.7^{ \pm 0.13}$ & $256 \underline{204}^{ \pm 24 \underline{20}}$ \\
\hline LU2 & $10.1^{ \pm 0.28}$ & $347^{ \pm 6}$ \\
\hline IN & $10.5^{ \pm 0.18}$ & $315 \underline{308} \underline{85}^{ \pm 5}$ \\
\hline
\end{tabular}

Table 8 : Mechanical properties of variants at $120^{\circ} \mathrm{C}$

\begin{tabular}{|c|c|c|}
\hline & E $(\mathbf{G P a})$ & $\boldsymbol{\sigma}_{\mathbf{m}}(\mathbf{M P a})$ \\
\hline LU1 & $1.6^{ \pm 0.18}$ & $54^{ \pm 3}$ \\
\hline LU2 & $2.6^{ \pm 0.15}$ & $143^{ \pm 12}$ \\
\hline IN & $3.2^{ \pm 0.45}$ & $187^{ \pm 20}$ \\
\hline
\end{tabular}

Table 9 : Mechanical tensile properties of variants at $150^{\circ} \mathrm{C}$

\begin{tabular}{|c|c|c|}
\hline & E (GPa) & $\boldsymbol{\sigma}_{\mathbf{m}}$ (MPa) \\
\hline LU1 & $2.7^{ \pm 0.23}$ & $41^{ \pm 4}$ \\
\hline LU2 & $2.9^{ \pm 0.17}$ & $97^{ \pm 4}$ \\
\hline IN & $3.4^{ \pm 0.6}$ & $90^{ \pm 9}$ \\
\hline
\end{tabular}




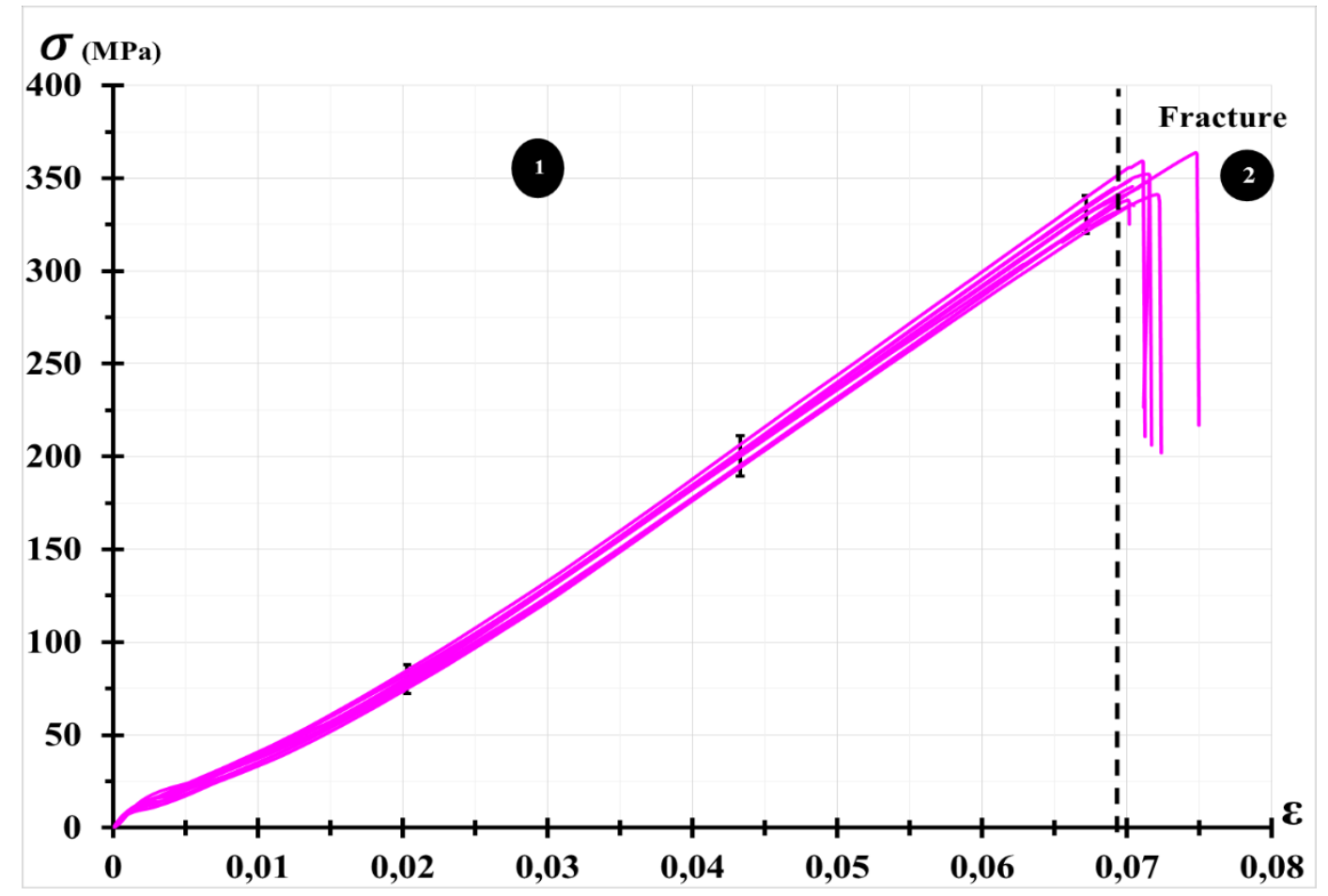

Fig. 3 : Tensile curves of LU2 at $\mathrm{T}_{\mathrm{amb}}$

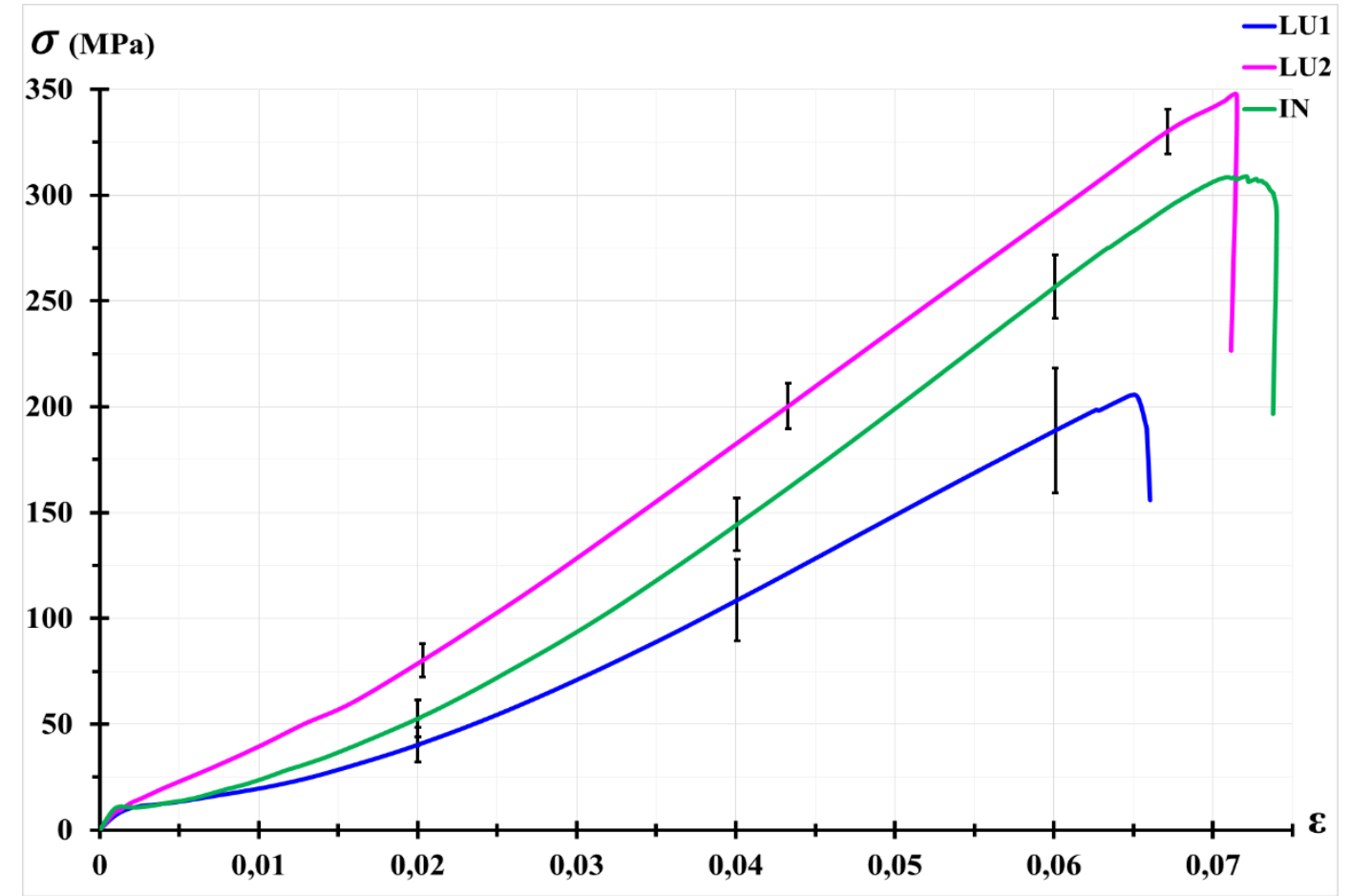

Fig. 4 : Tensile tests curves of different variants at $T_{a m b}$ 


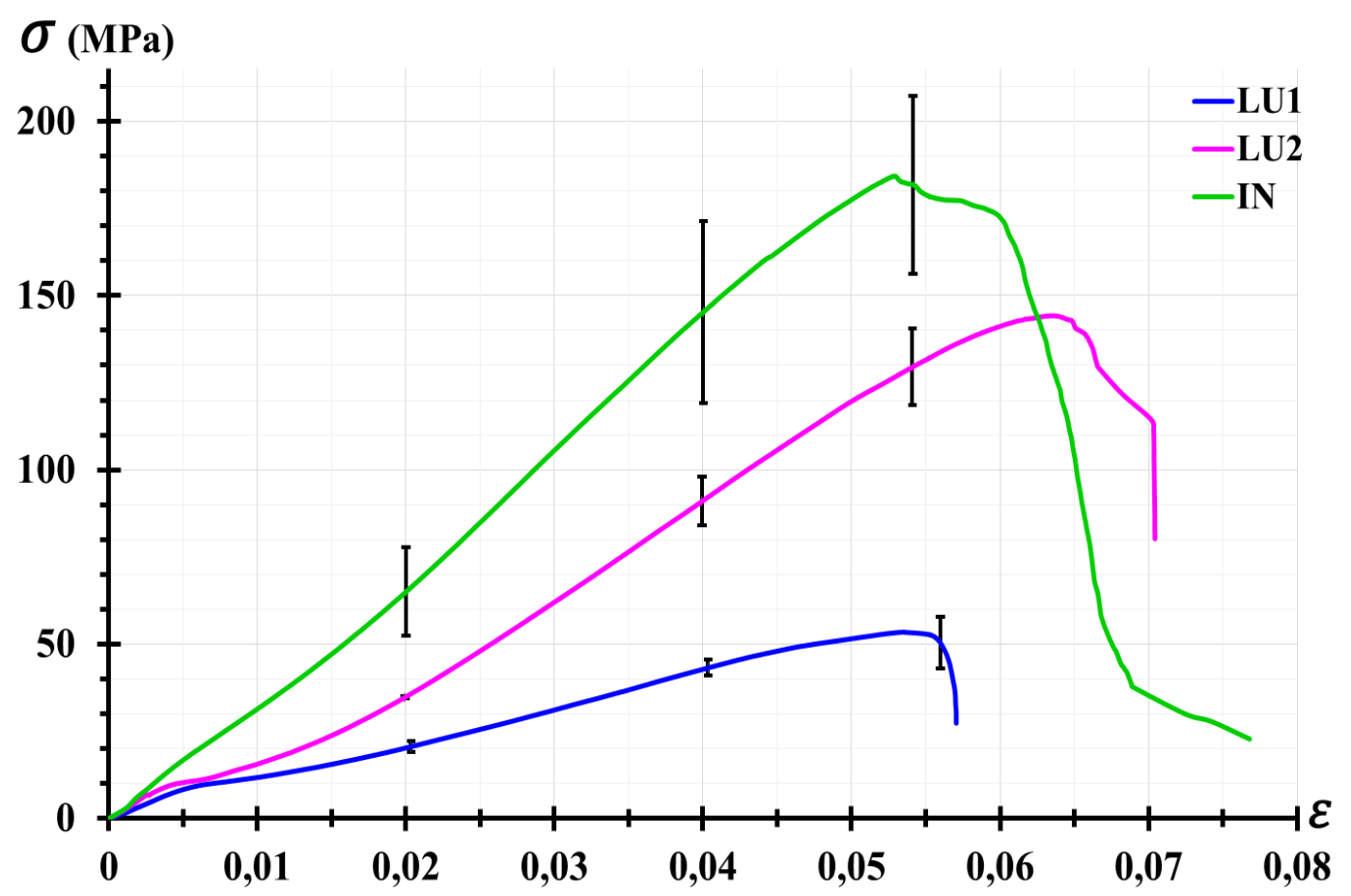

Fig. 5 : Tensile tests of different variants at $120^{\circ} \mathrm{C}$

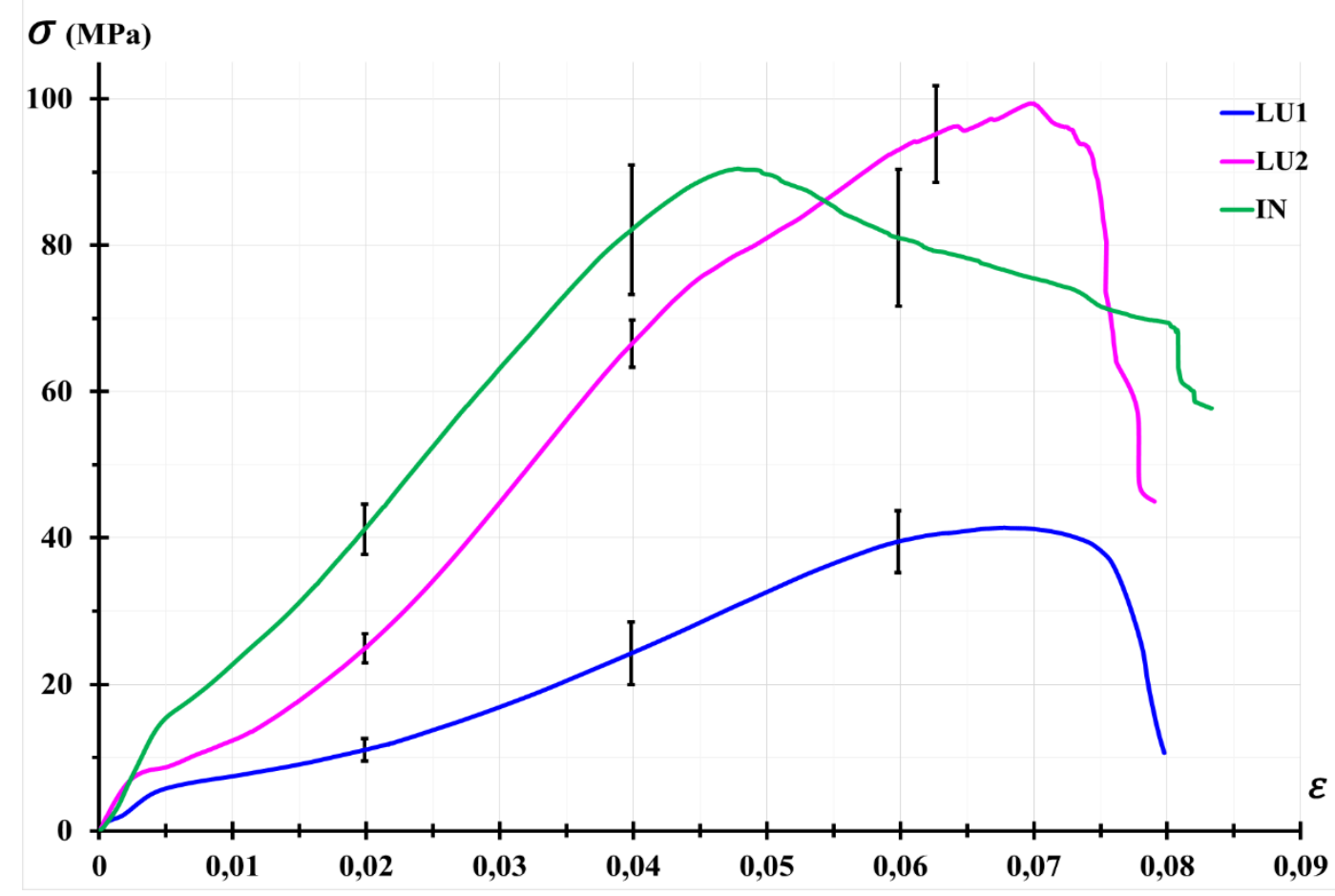

Fig. 6 : Tension curves at $150^{\circ} \mathrm{C}$ 


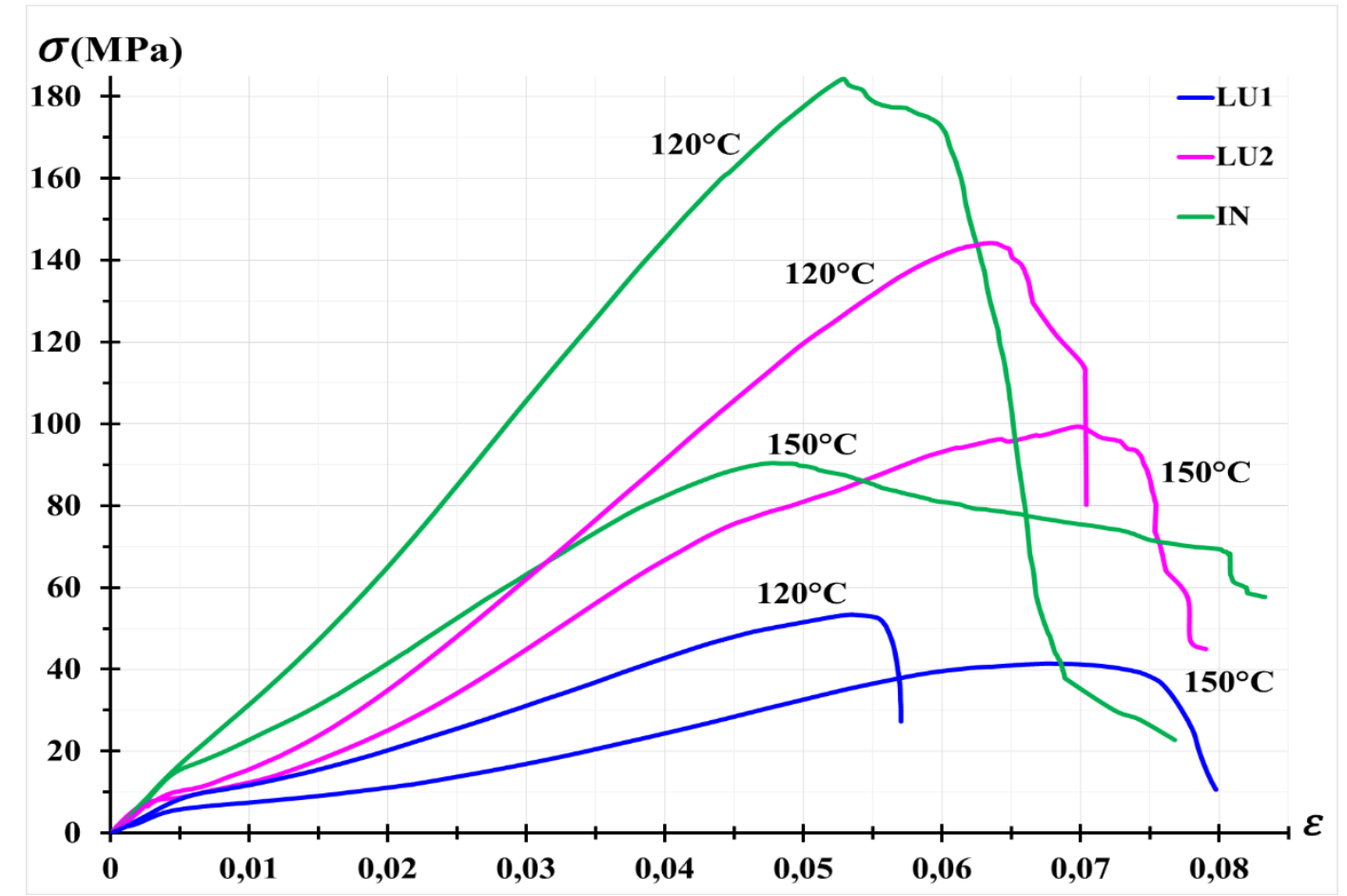

Fig. 7 : Tensile curves at $120^{\circ} \mathrm{C}$ and $150^{\circ} \mathrm{C}$

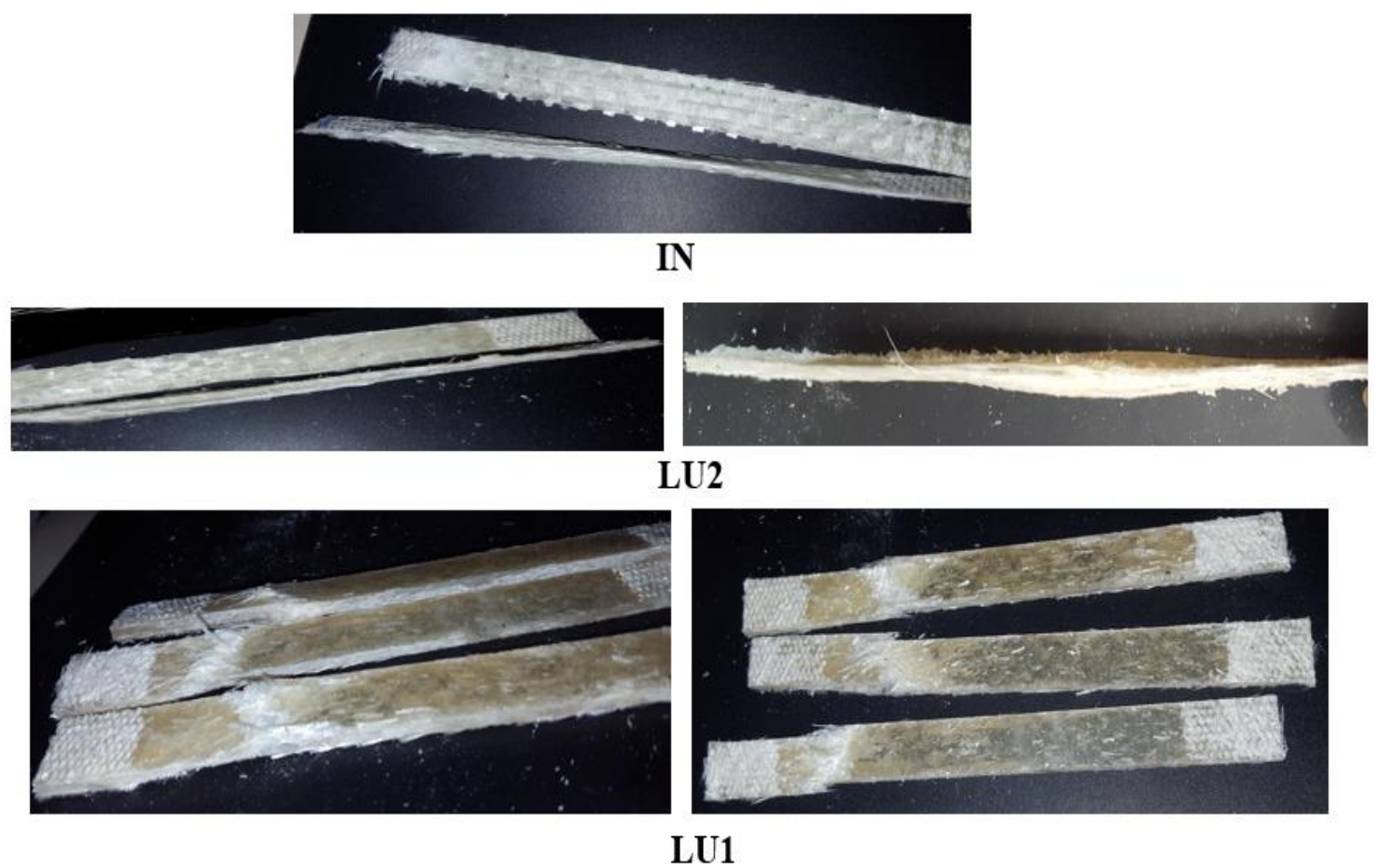

Fig. 8 : Tensile fracture modes at $120^{\circ} \mathrm{C}$ 


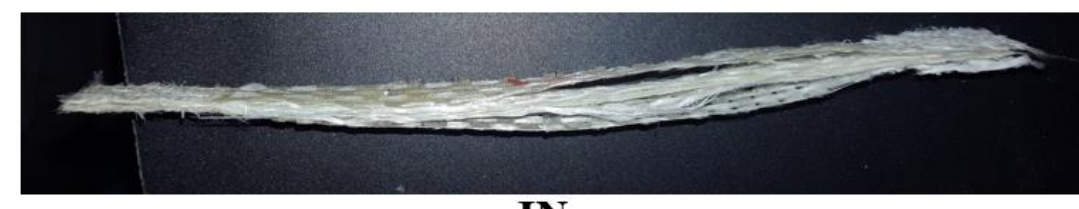

IN

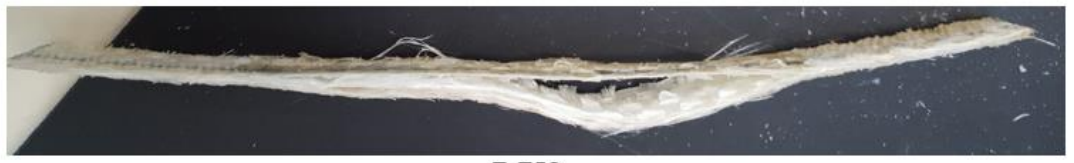

LU2

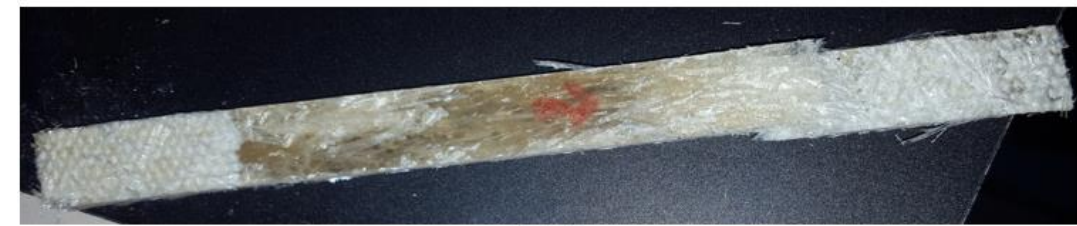

\section{LU1}

Fig. 9 : Tensile fracture modes at $150^{\circ} \mathrm{C}$

\subsubsection{Bending flexural test}

\section{$\checkmark \quad \operatorname{At~}_{\mathbf{a m b}}(\mathrm{V}=\mathbf{5 m m} / \mathbf{m i n})$}

Fig. 10 presents the average curves of different variants on 10 samples, for the 3 -points flexion at $\mathrm{T}_{\mathrm{amb}}$. These static tests show a quasi-rigid behavior for the 3 variants. The LU2 and IN variants remain stiffer than the LU1 variant even in flexion. The IN variant is more resistant than the LU2 variant, in flexural test. It has the highest Young's modulus in bending (Fig. 11).

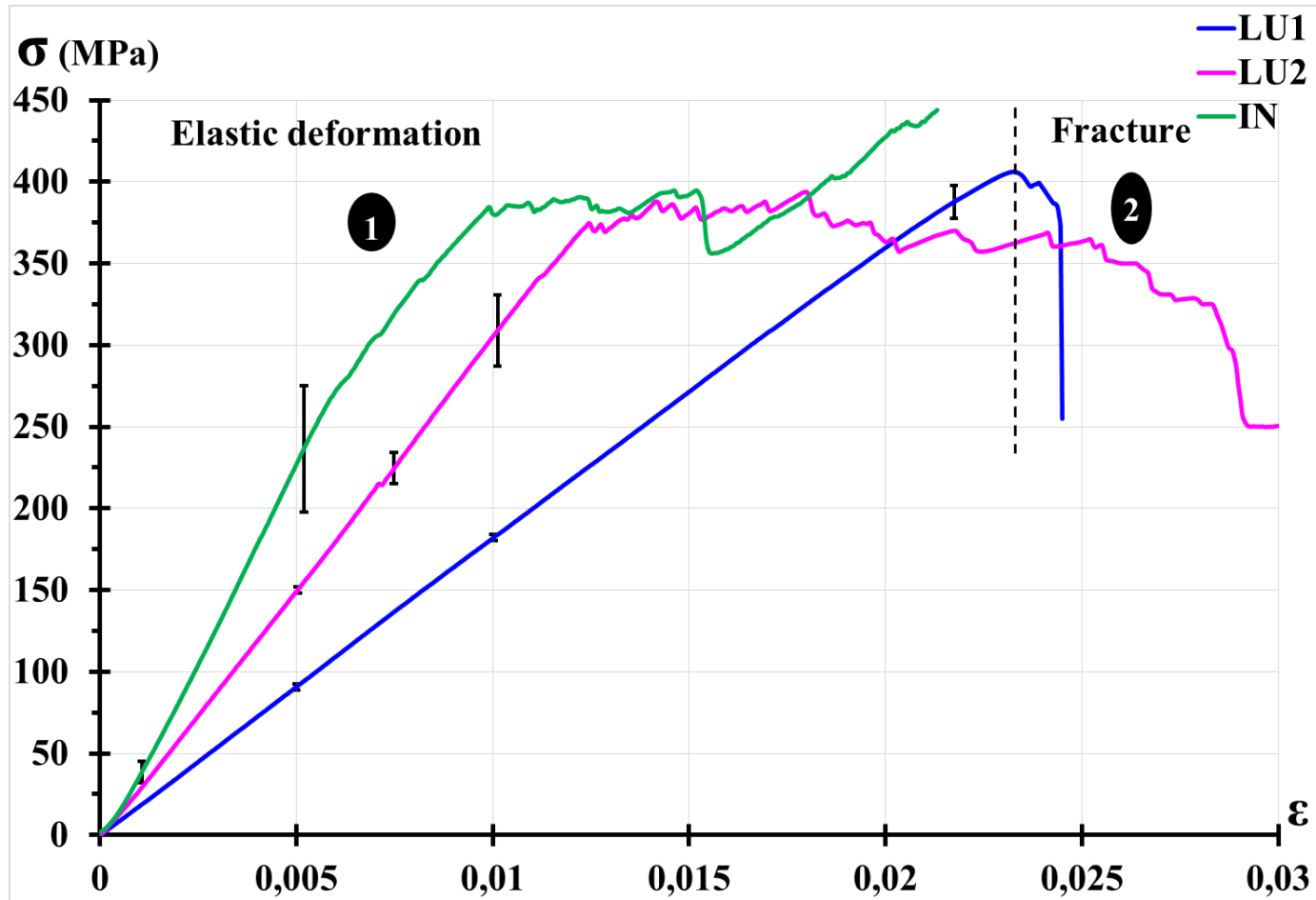

Fig. 10 : Bending curves at $\mathrm{T}_{\mathrm{amb}}$ of different variants 


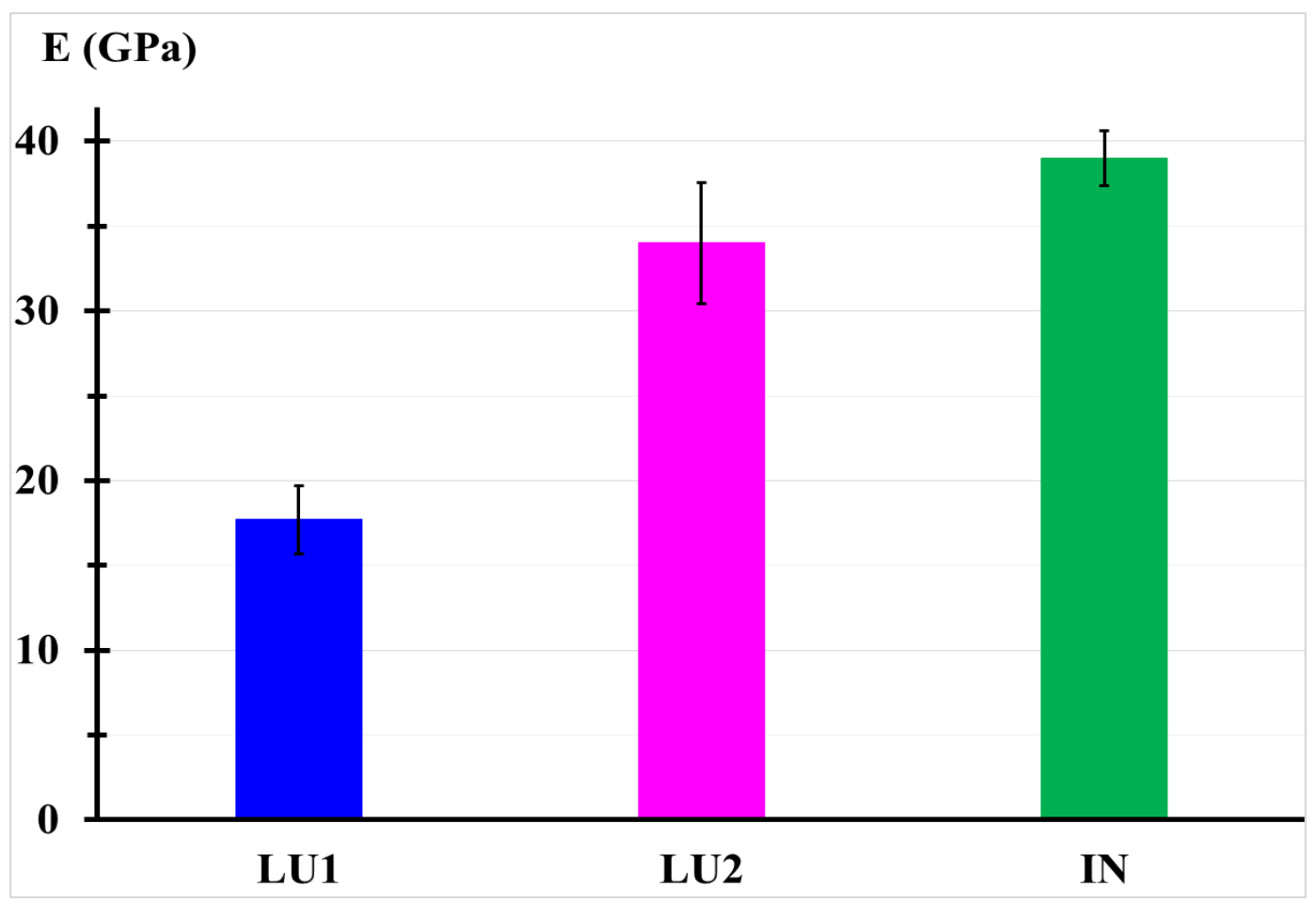

Fig. 11 : Average Young's modulus of different variants

\subsection{Dynamic study: Dynamic bending}

One of the difficulties of the thermo-mechanical study of the composite is its composition. This material is heterogeneous due to the presence of the different phases such as glass fibers, resin, fillers and arrangement of its phases and orientation of its fibers. So, to understand the effect and the impact of its microstructures on its properties, we need to treat and to analyze the Young's modulus variation as a function of temperature and to characterize the dynamic behavior of our materials.

Fig.12 shows a representative curve of dynamic 3-point bending test of variant LU2, which has three main areas:

Glassy zone: the composite remains in its glassy state. Its modulus decreases slowly with the increase of temperature and is in the order of $8 \mathrm{GPa}$. This high rigidity results from the immobility of chains. This state is characterized by a very low deformability.

Glassy / rubbery transition: the elastic modulus decreases with the increase of the temperature. It is the glass transition temperature, $\mathrm{T}_{\mathrm{g}}$, zone. When the temperatures increase, motions of segments of the molecular chains are allowed so, the Young's modulus decreases. Therefore, the increase (or decrease) of the temperature results in a significant decrease (or increase) of the stiffness of the material and therefore its Young's modulus [18]. This decrease could be the Payne effect [19, 20] (explained in next paragraph, Fig. 13).

In addition, this zone shows the transition of the material from the fragile state to a rubbery state characterized by a higher deformability.

Rubber zone: the material is in its rubbery state. In this zone, the elastic modulus is nearly constant. This can be explained by the domination of the thermal agitation over the interactions between the chains and the elastic modulus becomes directly linked to the crosslinking (chemical bonds) and to the entanglement of the chains (physical bonds, hydrodynamic effect) [20]. However, it is noted that the cooling curve does not follow the same path as the heating curve. This indicates the presence of the hysteresis phenomenon during this test for the LU2 variant. 


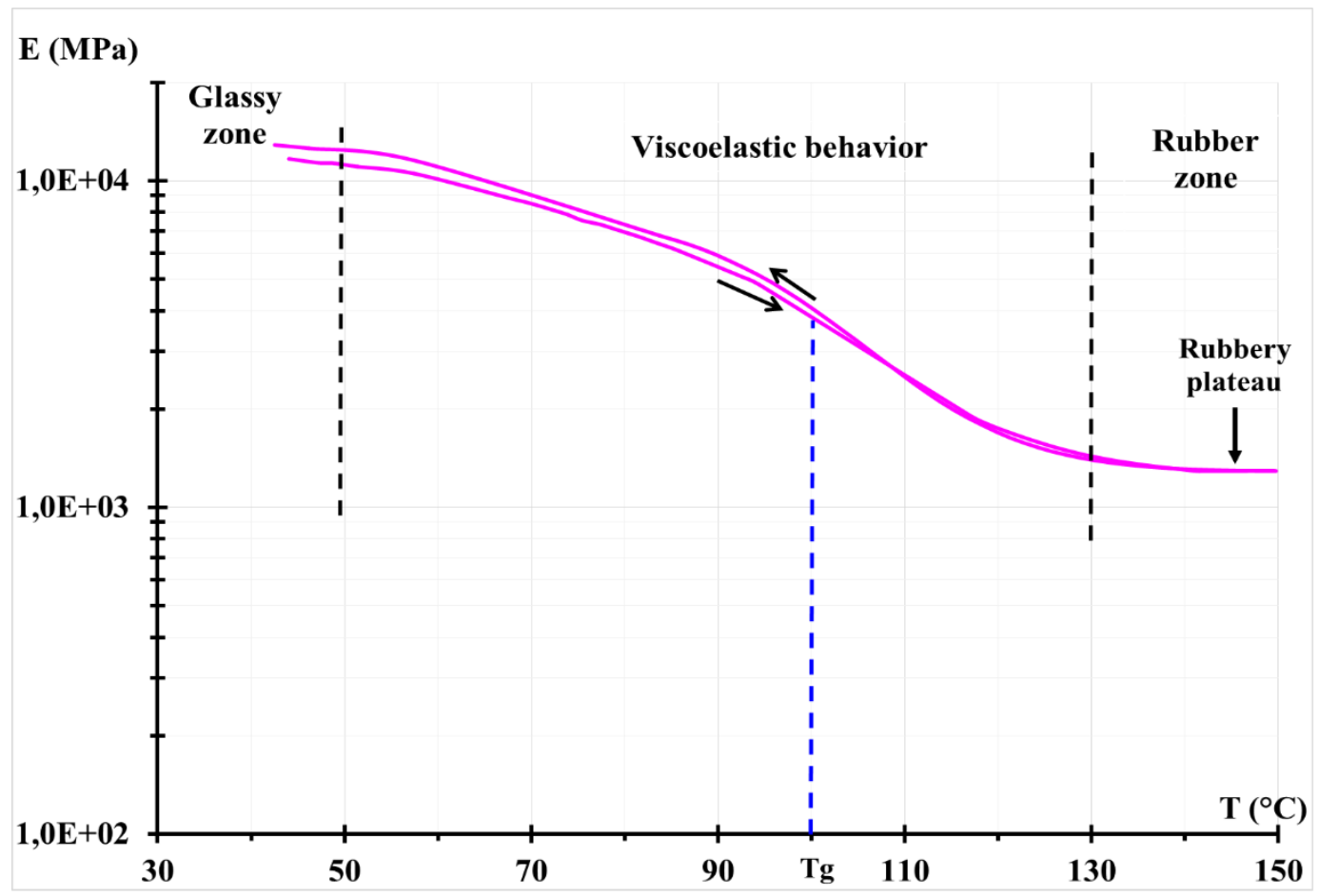

Fig. 12 : Representative curve of dynamic bending test of LU2

For Payne effect, a dynamic bending flexural test is done for $\mathrm{LU} 1$ at $150^{\circ} \mathrm{C}$, while varying dynamic displacement between $9.10^{-5} \mathrm{~m}$ and $4.10^{-4} \mathrm{~m}$. We have chosen $150^{\circ} \mathrm{C}$ because at this temperature, our material is in its rubber state. The frequency is around $1 \mathrm{~Hz}$ and the static displacement is $6.10^{-4} \mathrm{~m}$. The choice of this frequency and static displacement is made after numerous tests, because these two conditions ensure the stability of the measurements. The dimensions of specimens are: $(45 \times 9.5 \times 3.2) \mathrm{mm}^{3}$. Fig. 13 shows the result of this test. The decrease of the modulus with the amplitude of the deformation is known as the Payne effect [19], well known by the filled rubber community. We observe that conservation Young modulus E' decreases with the increase of the strain which could be contributed to the loss of the rigidity of composite. The Young's modulus E' decreases while the loss factor tan $\delta=E " / E$ ' increases as a function of strain. All these observations justify the presence of Payne effect.

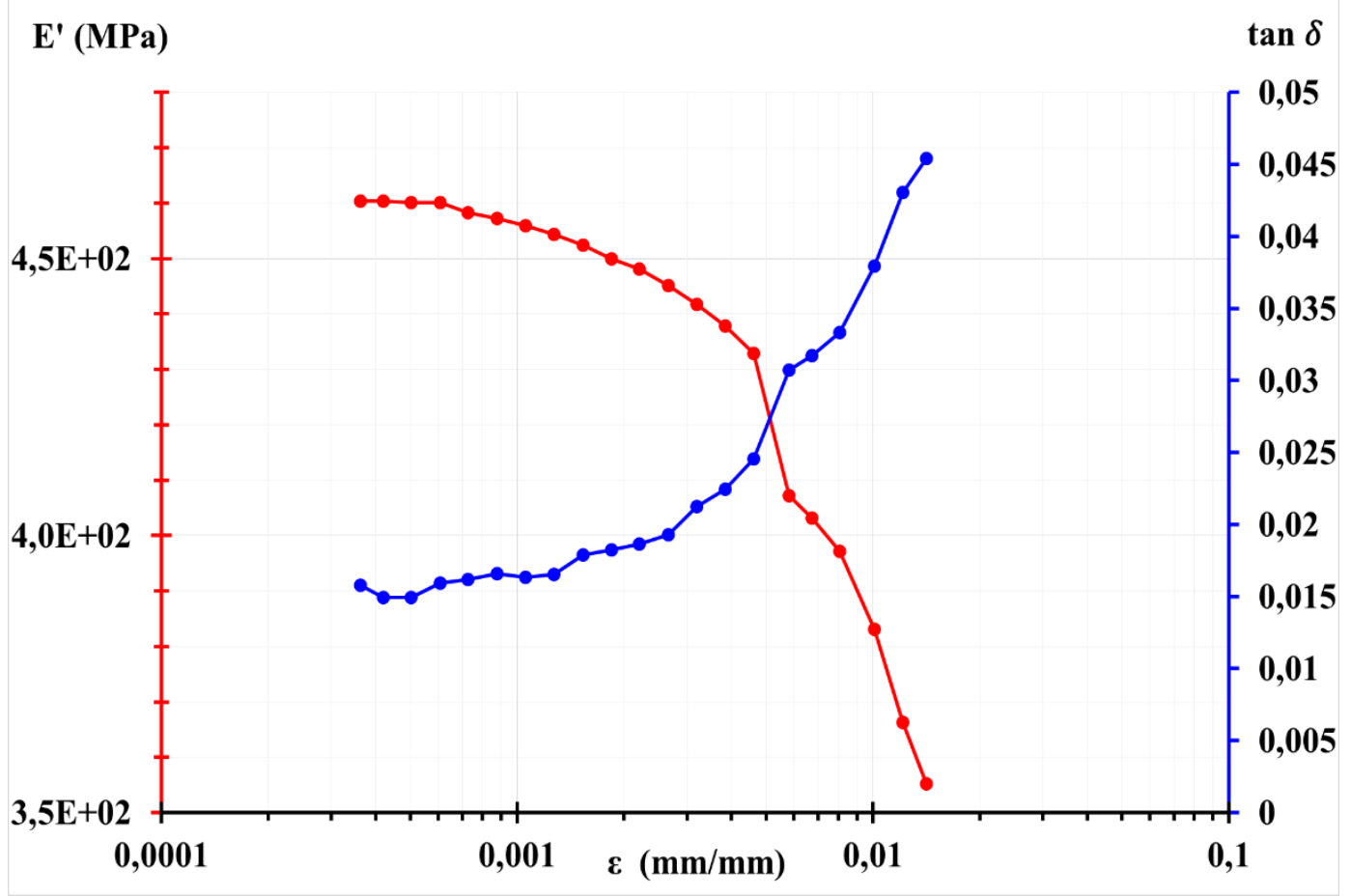

Fig. 13 : Dynamic bending of LU1 


\section{$\checkmark$ Dynamic bending of different composites}

For $20^{\circ} \mathrm{C} \leq \mathrm{T} \leq 150^{\circ} \mathrm{C}$, figures 14 and 15 show the evolution of Young's modulus and loss factor as a function of temperature. According to the definition given in literature [21], an unstable behavior is revealed when the elastic modulus curve does not follow the same path in cooling and in heating, showing a likely physical or chemical change at high temperatures. In our case, since the samples were cured according to the $5^{\text {th }}$ cycle, a post curing is unlikely. Following [12], some other crosslinking reactions might have started at $150^{\circ} \mathrm{C}$. It is also obvious that the glass transition temperature of the infusion resin is higher than the hand lay-up resin one

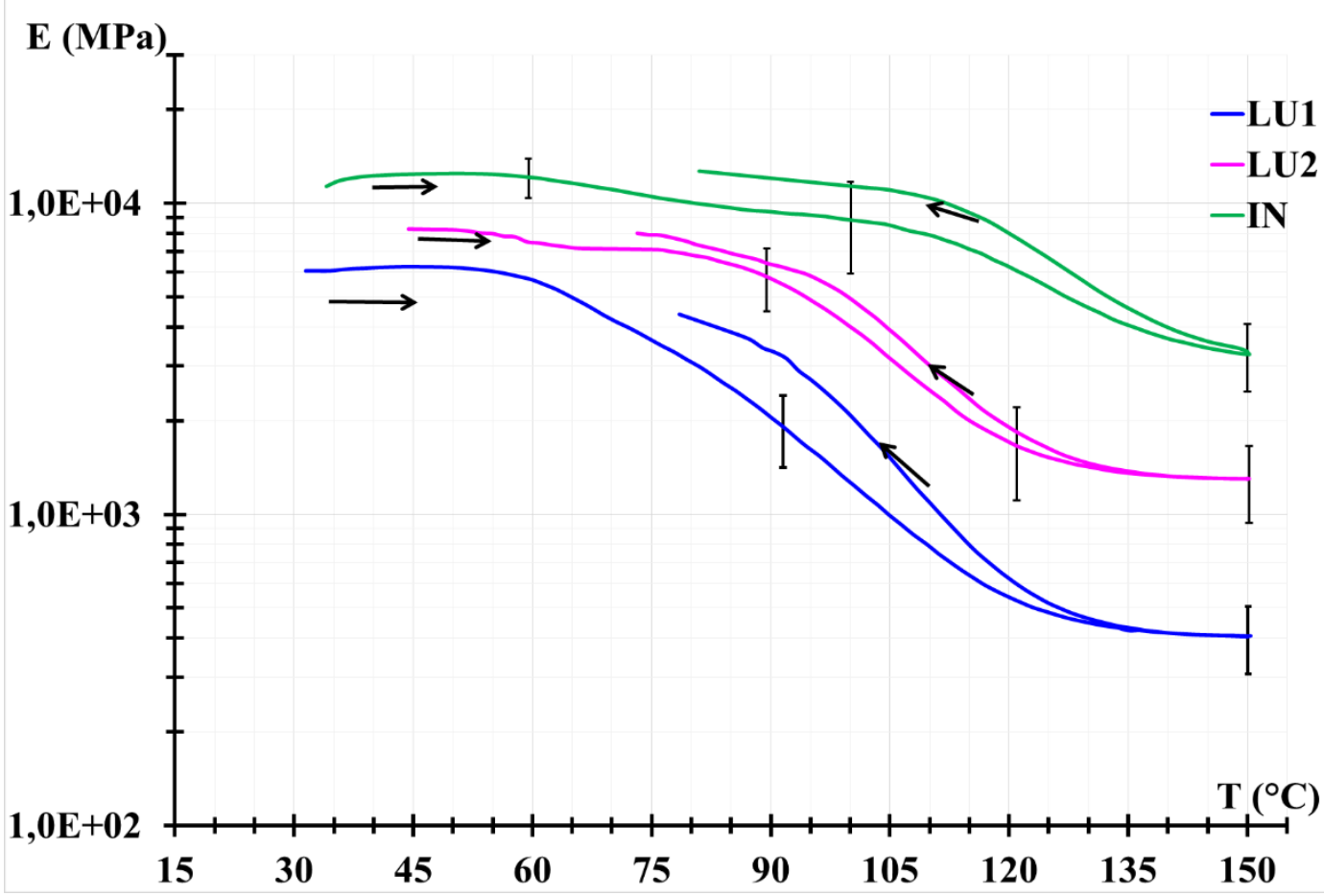

Fig. 14 : Dynamic bending of different variants

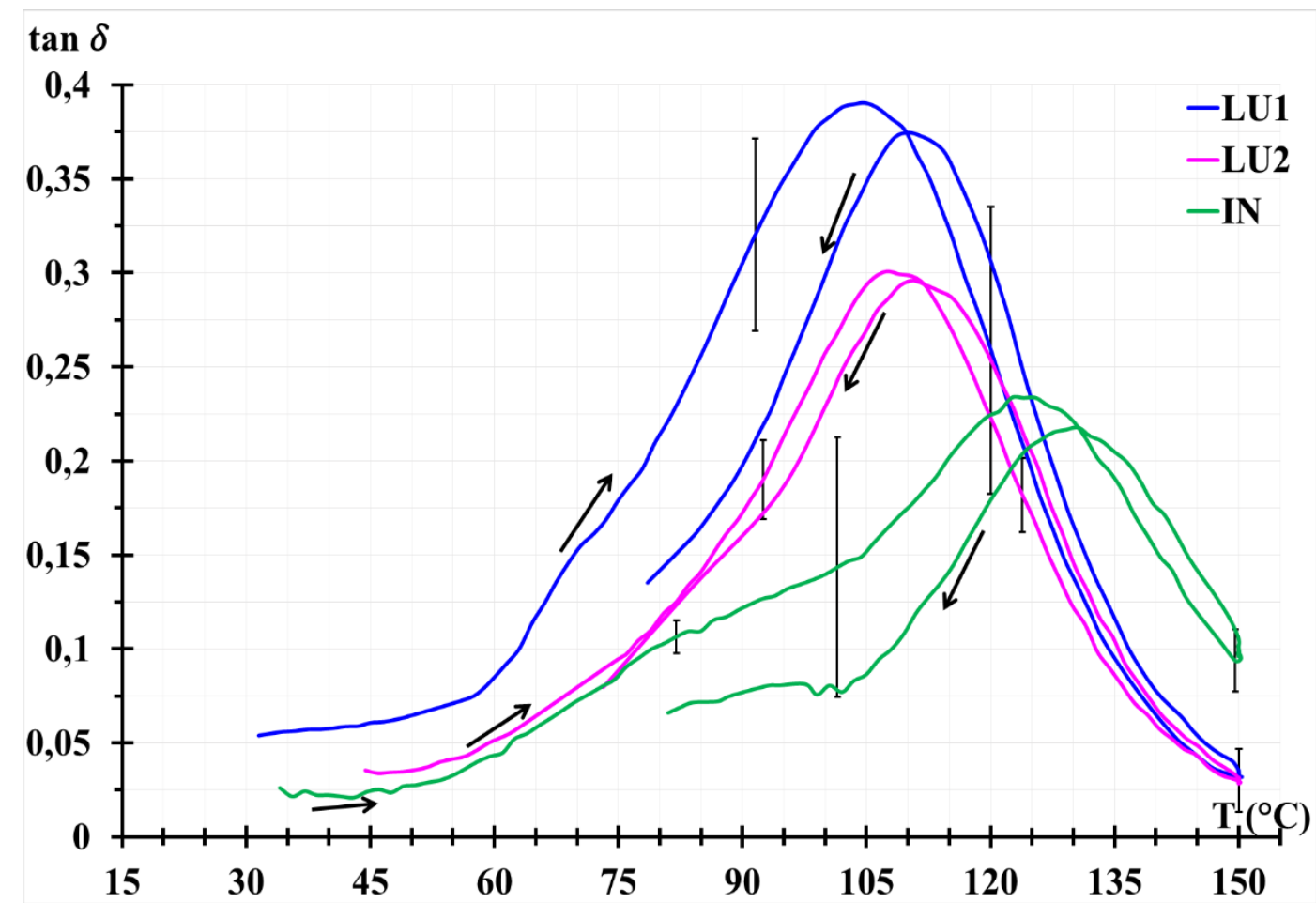

Fig. 15 : Average $\tan \delta$ of different variants 


\section{$\checkmark$ Comparison of the Young's modulus of different composites in bending at high temperatures}

The variant IN presents the highest Young's modulus in dynamic bending at different temperatures, as shown in Fig.16. The composite-infusion has higher fibers content than the other composites, so that's why its Young's modulus is higher. Pyrolysis tests were done for LU2 and for IN specimens to verify the fibers volume ratio. It was found that the fibers volume ratio for IN is between $64 \%-66 \%$ and for LU2, it has varied between $51 \%$ and $53 \%$.

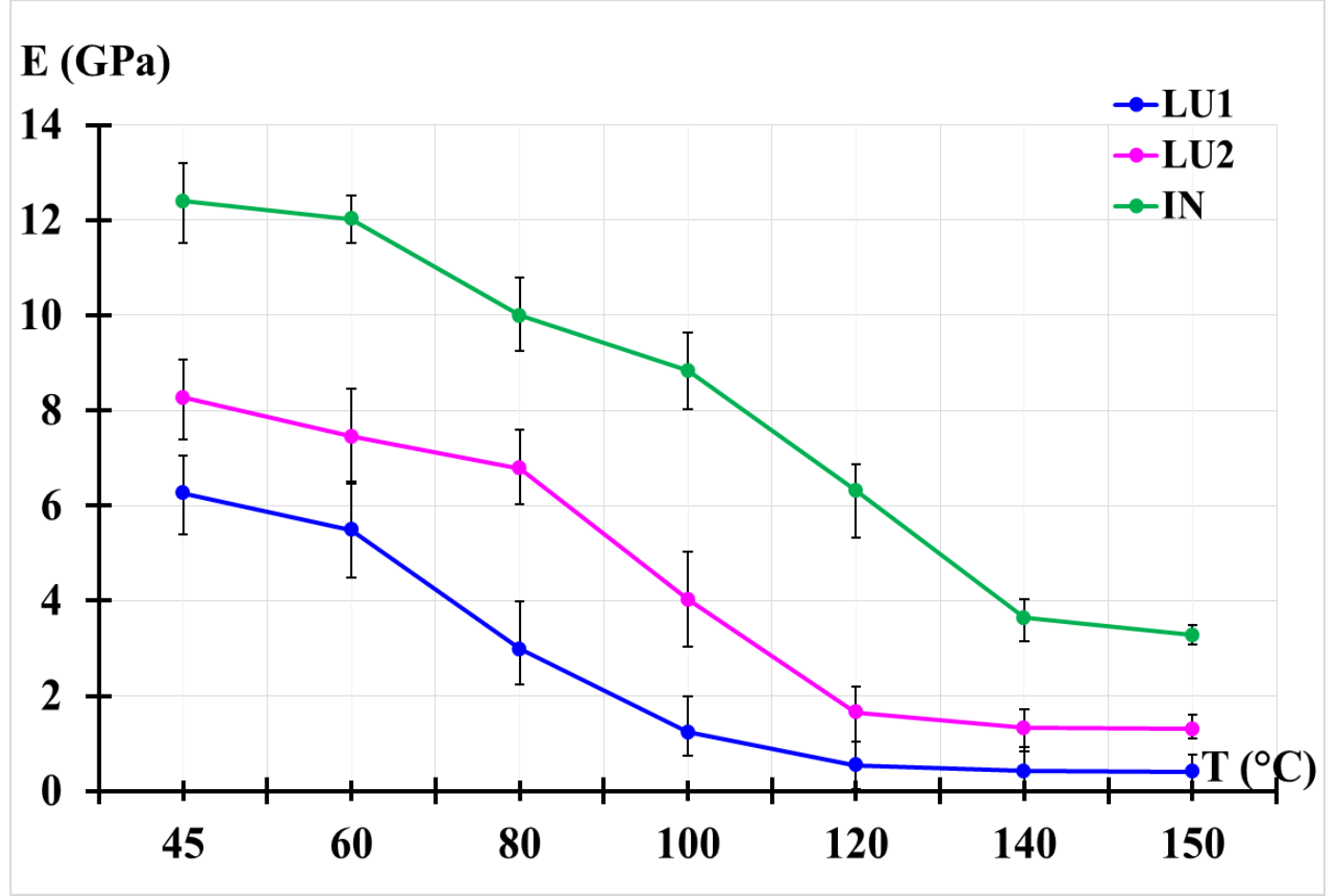

Fig. 16 : Average Young's modulus of different variants

\subsection{Study of composite ageing}

\subsubsection{Mechanical ageing: Cyclic tensile of variant LU1}

Since the temperatures used in the recycling processes may reach $150^{\circ} \mathrm{C}$, it is of some interest to characterize the composite at this temperature. A cyclic loading is relevant as in the processing conditions, loading and unloading are likely to occur. Under these conditions, the composite is in its rubbery state. Fig. 17 presents the first type of cyclic loading imposed for $\mathrm{LU} 1$ at $150^{\circ} \mathrm{C}$, while varying the strain between $0 \%$ and $3 \%$. The result (Fig. 18) shown an important drop of stress from the $1^{\text {st }}$ cycle to the $2^{\text {nd }}$ one. This shows a drop of the modulus of the material. Also, a decrease of the secant modulus measured from the origin (0 (strain), 0 (stress)), is observed for each loading. This behavior reflects the Mullins effect [22-24].

The unloading did not follow the same path as the loading: it is the phenomenon of hysteresis [23, 25]. Thus, the cyclic behavior of this composite can be assimilated to the behavior of the elastomers which is characterized by the hysteresis loop. 


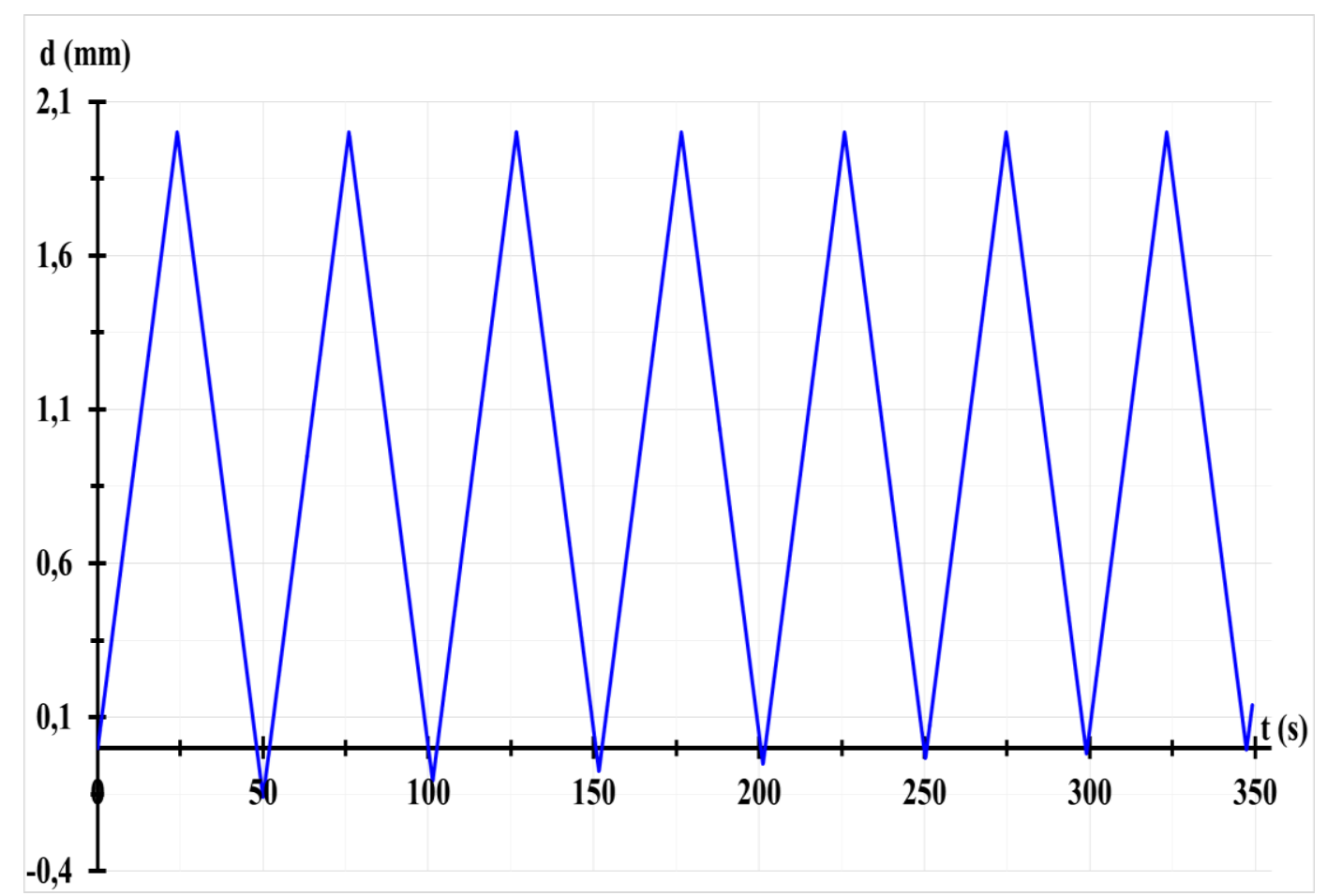

Fig. 17 : Cyclic loading imposed for LU1 at $150^{\circ} \mathrm{C}$

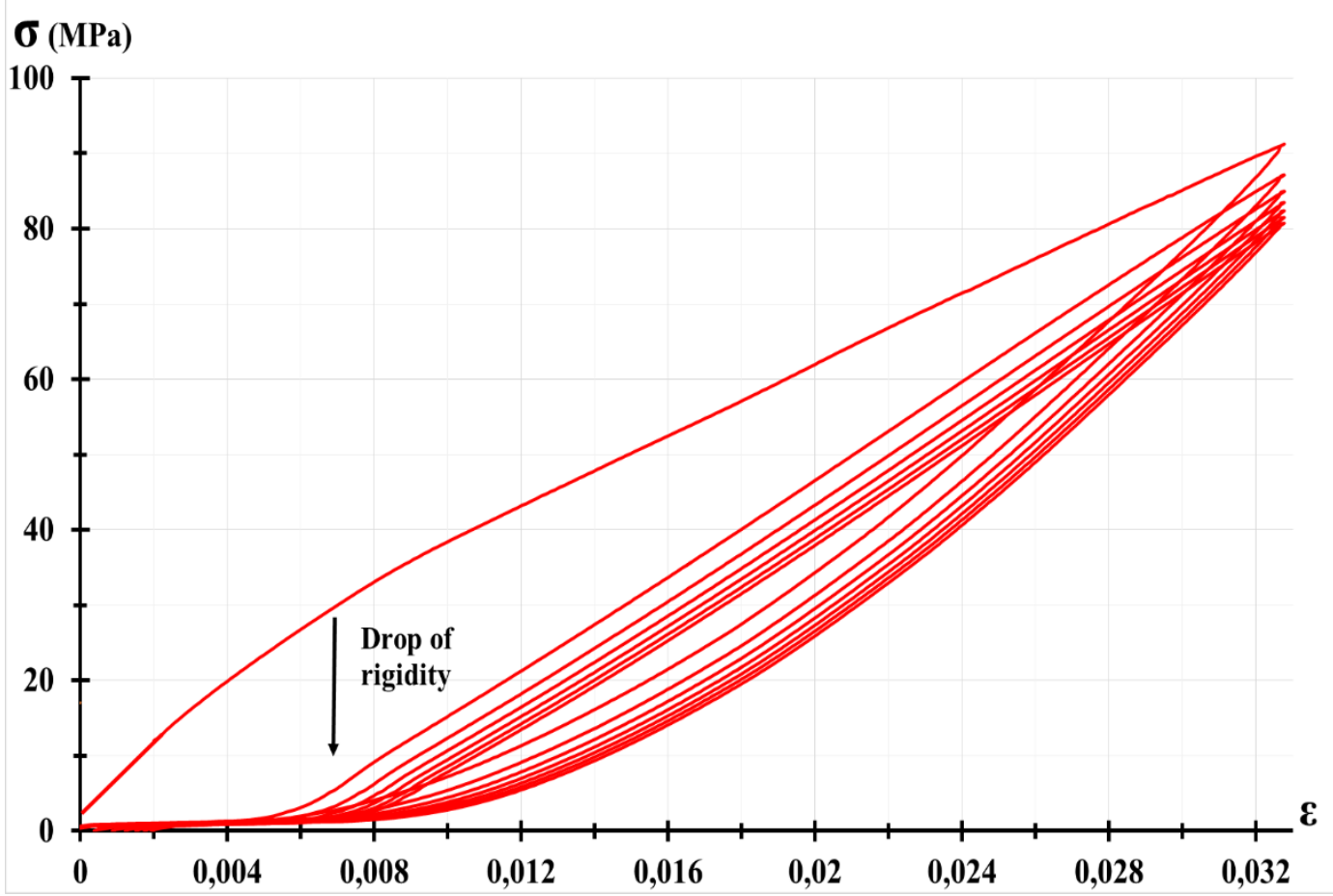

Fig. 18 : Result of the cyclic tensile test of LU1 at $150^{\circ} \mathrm{C}$

Fig. 19 shows another type of cyclic loading with imposed displacement for specimens LU1. It is characterized by the rise of the maximum strain level with the number cycle and a return to zero stress. The results, presented Fig. 20, show hysteresis loops and softening. So, the same phenomena are observed at different strain levels.

To better understand the behavior of our material while submitted to different levels of imposed loading, another type of loading presented in Fig.21, has been imposed. It involves cyclically soliciting the composite sample at different strain levels and at each strain level, we perform 5 cycles. The return is set at 0 stress. 
After applying the selected loading type, figures 22 and 23 show the results of stress as a function of strain and of time. We observe at the same level of imposed displacement, a decrease of the stress, which shows a drop of stiffness. Also, this decrease of stress has the same shape (for these 5 cycles) while varying the strain level (Fig. 23). The same phenomena were noticed at each strain level showing a Mullins effect. So, in our case of unsaturated polyester glass fibers reinforced composite, the following phenomena could be noticed during cyclic loading at $150^{\circ} \mathrm{C}$ : hysteresis, Mullins effect and stress relaxation. Above its glass transition temperature, this composite shows features of rubber materials, which is expected from soft amorphous phase.

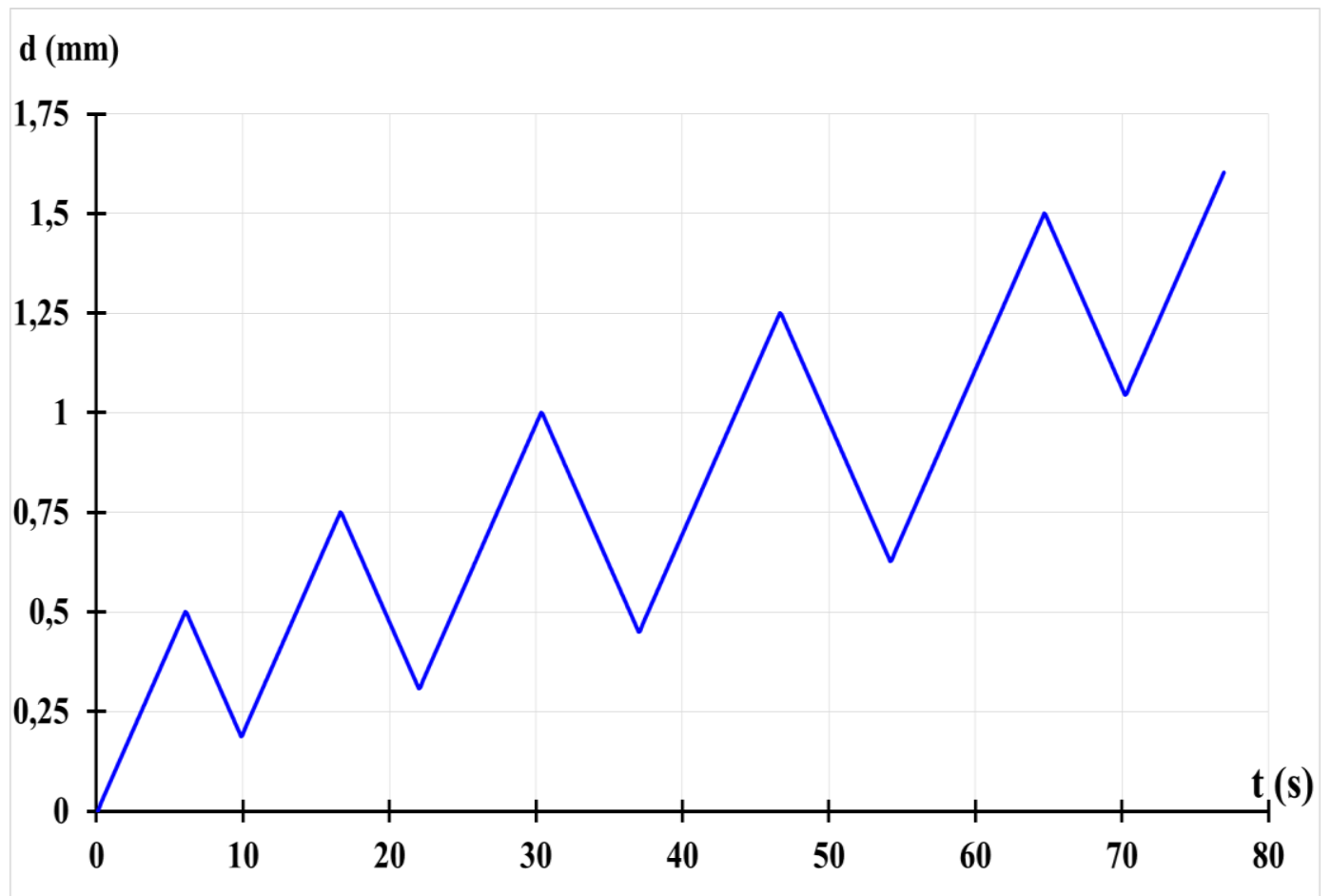

Fig. 19 : Cyclic loading imposed for LU1 at $150^{\circ} \mathrm{C}$

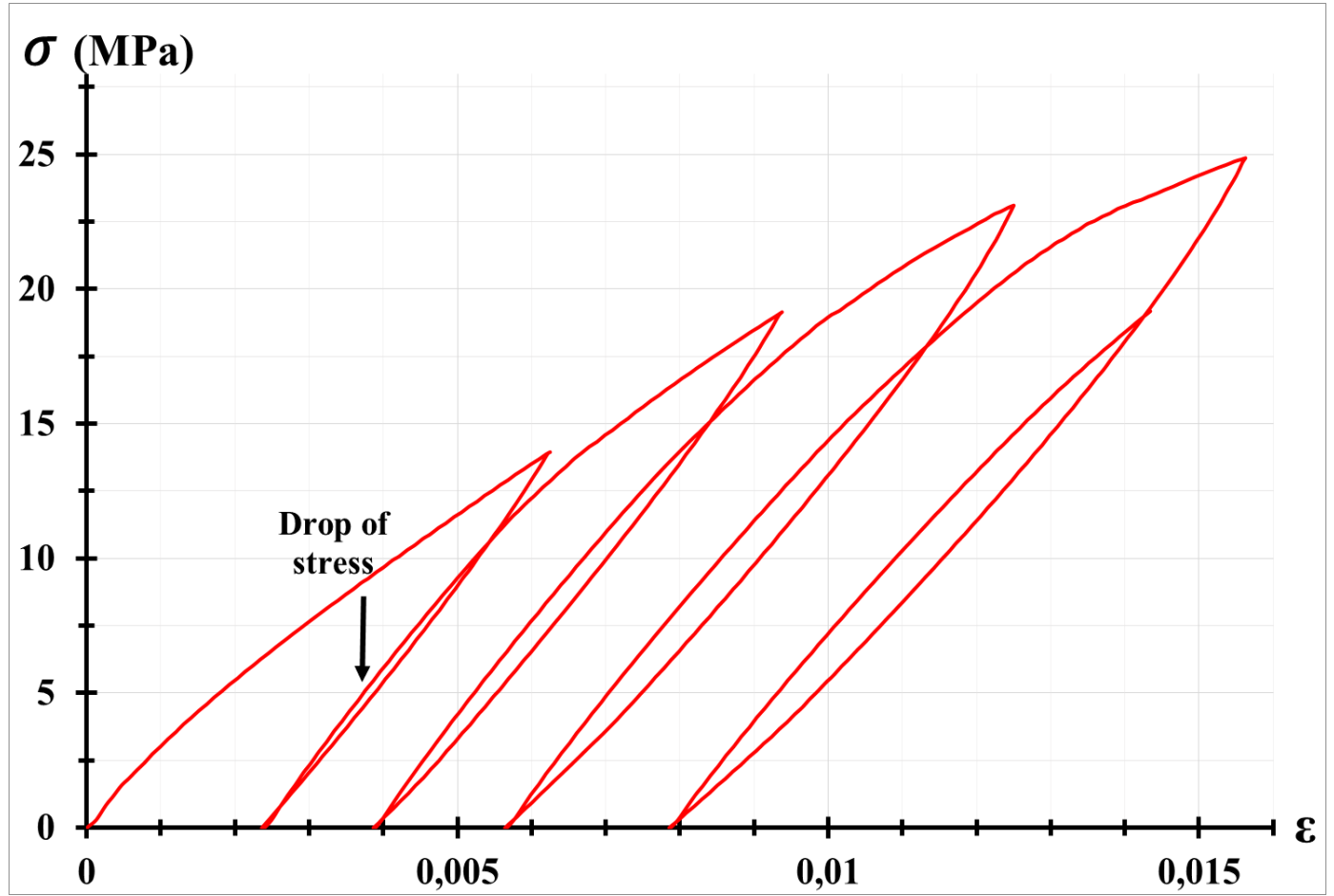

Fig. 20 : Result of the cyclic tensile test of LU1 at $150^{\circ} \mathrm{C}$ 


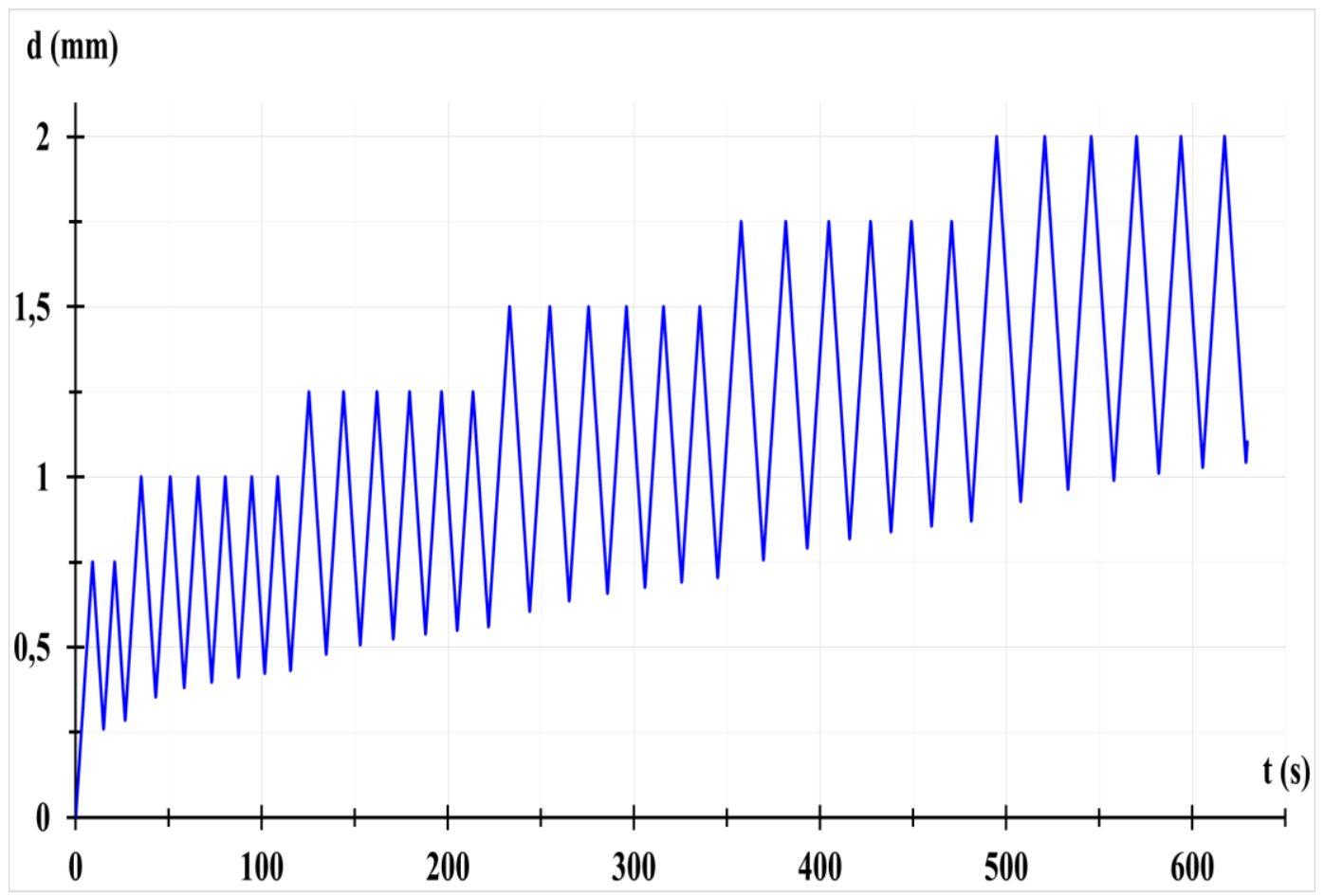

Fig. 21 : Cyclic loading imposed to LU1 at $150^{\circ} \mathrm{C}$

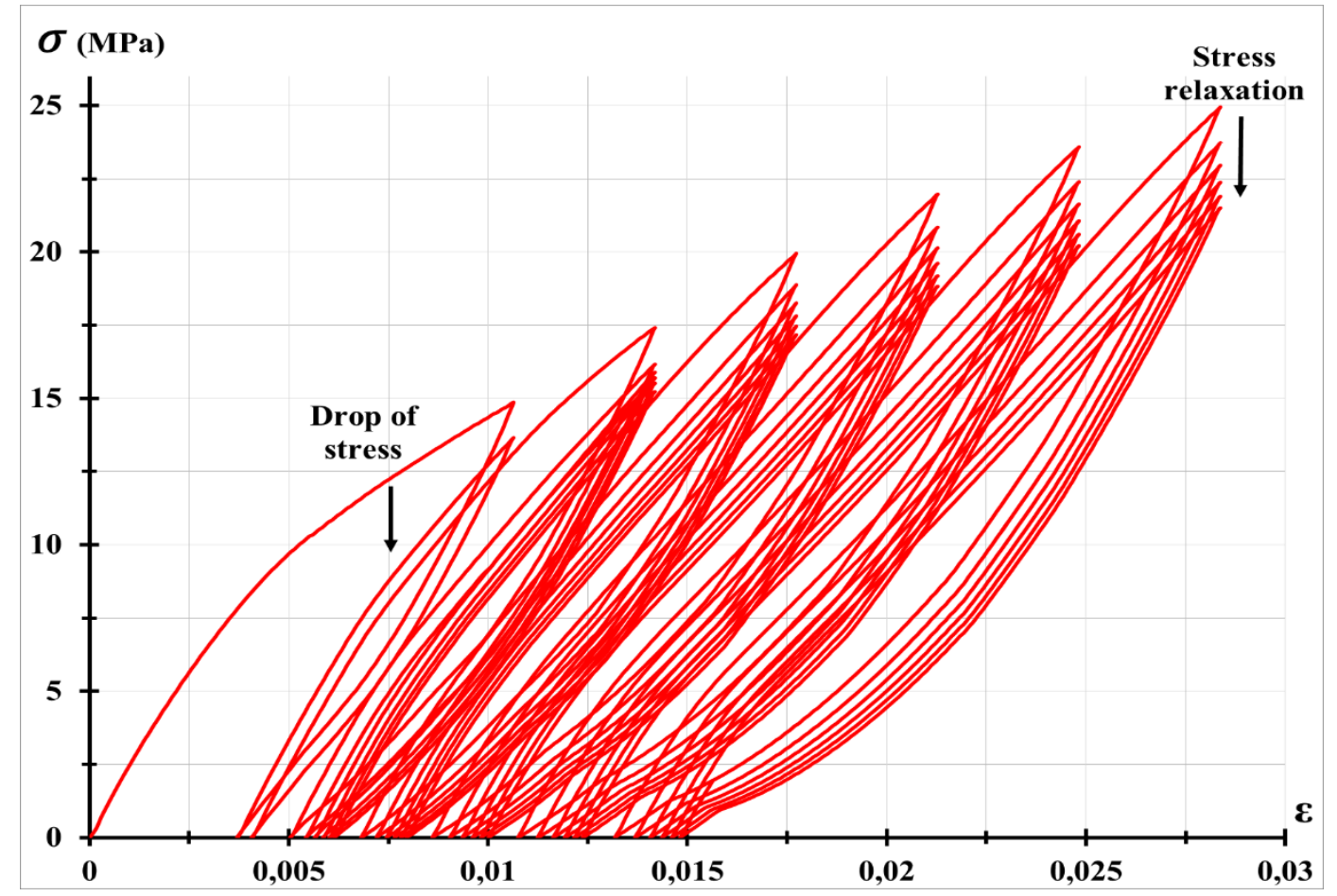

Fig. 22 : Result of the cyclic test of LU1 at $150^{\circ} \mathrm{C}$ 


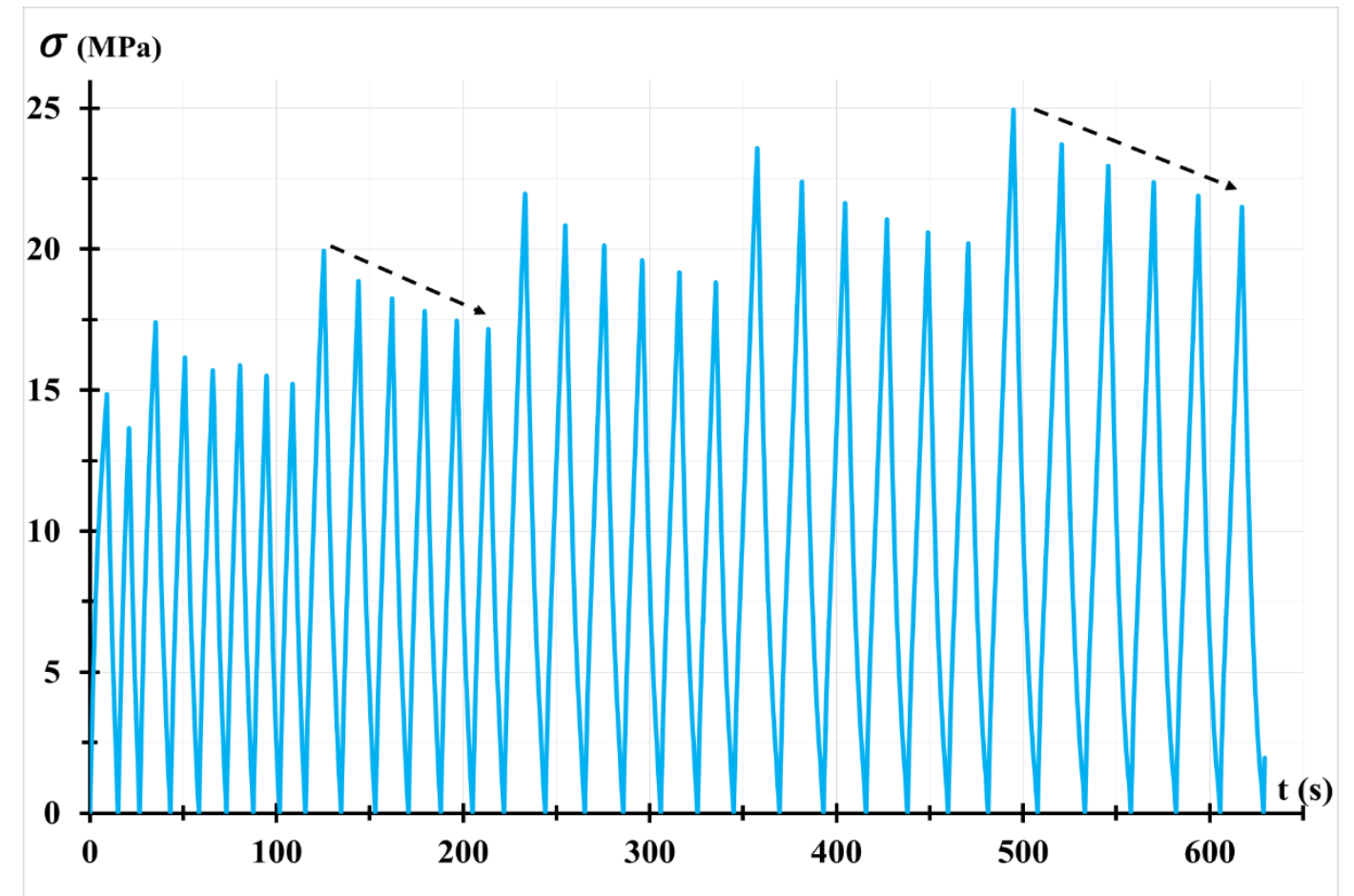

Fig. 23 : Result of the cyclic tensile test variant LU1 at $150^{\circ} \mathrm{C}$

\subsubsection{Thermochemical ageing: hydrolysis of LU1}

The table 10 displays the water saturation rates of each protocol test described in paragraph 2.3.2/Thermomechanical behavior ageing, after hydrolysis and after drying, just before the 3 points flexural bending test.

Table 10 : Water saturation rate of composite LU1 of all protocols tests of hydrolysis

\begin{tabular}{|c|c|c|c|c|c|c|c|}
\hline & & \multicolumn{2}{|c|}{$1^{\text {st }}$ protocol at Tamb } & \multicolumn{2}{|c|}{$2^{\text {nd }}$ protocol at $80^{\circ} \mathrm{C}$} & \multicolumn{2}{|c|}{$3^{\text {rd }}$ protocol at $90^{\circ} \mathrm{C}$} \\
\hline \multirow{2}{*}{\multicolumn{2}{|c|}{$\begin{array}{c}\text { Water } \\
\text { saturation } \\
\text { rate } S_{t} \\
(\%)\end{array}$}} & $\begin{array}{c}\text { End of } \\
\text { hydrolysis }\end{array}$ & Before test & $\begin{array}{c}\text { End of } \\
\text { hydrolysis }\end{array}$ & Before test & $\begin{array}{c}\text { End of } \\
\text { hydrolysis }\end{array}$ & $\begin{array}{c}\text { Before } \\
\text { test }\end{array}$ \\
\hline & & $\mathbf{S}_{\mathbf{t}}$ & $\mathbf{S}_{\mathbf{t}}$ & $\mathbf{S}_{\mathrm{t}}$ & $\mathbf{S}_{\mathrm{t}}$ & $\mathbf{S}_{\mathrm{t}}$ & $\mathbf{S}_{\mathbf{t}}$ \\
\hline \multirow{7}{*}{$\frac{\mathscr{E}}{\bar{E}}$} & Sp 1 & 2,63 & 1,93 & 3,15 & 1,15 & 2,47 & 1,08 \\
\hline & Sp 2 & 2,5 & 1,97 & 3,21 & 1,12 & 2,26 & 0,69 \\
\hline & Sp 3 & & & 3,32 & 1,1 & 1,95 & 0,85 \\
\hline & Sp 4 & & & 3,07 & 0,97 & 1,98 & 0,92 \\
\hline & Sp 5 & & & 3,07 & 0,14 & & \\
\hline & Sp 6 & & & 3,17 & 0 & & \\
\hline & Sp 7 & & & 3,15 & 0 & & \\
\hline
\end{tabular}

Fig. 24 presents the curves of the water absorption of samples while hydrolysis at $\mathrm{T}_{\mathrm{amb}}$. We notice that the sample Sp1 achieved its total water saturation rate after 500 hours in distillated water but sample Sp 2 achieved its total water saturation after 576 hours. This shift could be due to their fractions volume of glass fibers which are close but they are not the same. The quasi linear relation between weight gain and square root of time indicates a simple diffusion phenomenon.

Fig. 25 shows the results of the $1^{\text {st }}$ test protocol of the 3 points bending flexural test of LU1 at $T_{\text {amb }}$ before hydrolysis (blue curves for unsaturated sp, virgin samples) and after hydrolysis at $\mathrm{T}_{\mathrm{amb}}$ and drying (green and red curves with $1,97 \%$ and $1,39 \%$ water saturation rates). There is still a significant amount of water left in the composite which may act as a plasticizer. We remark that the hydrolyzed samples at $\mathrm{T}_{\mathrm{amb}}$ lose $66 \%$ of their 
flexural resistance ( 149 MPa) compared to virgin samples ( $450 \mathrm{MPa})$ but they get a gain of $195 \%$ in their deformation ration. So, that confirmed that the water plays the role of plasticizer.

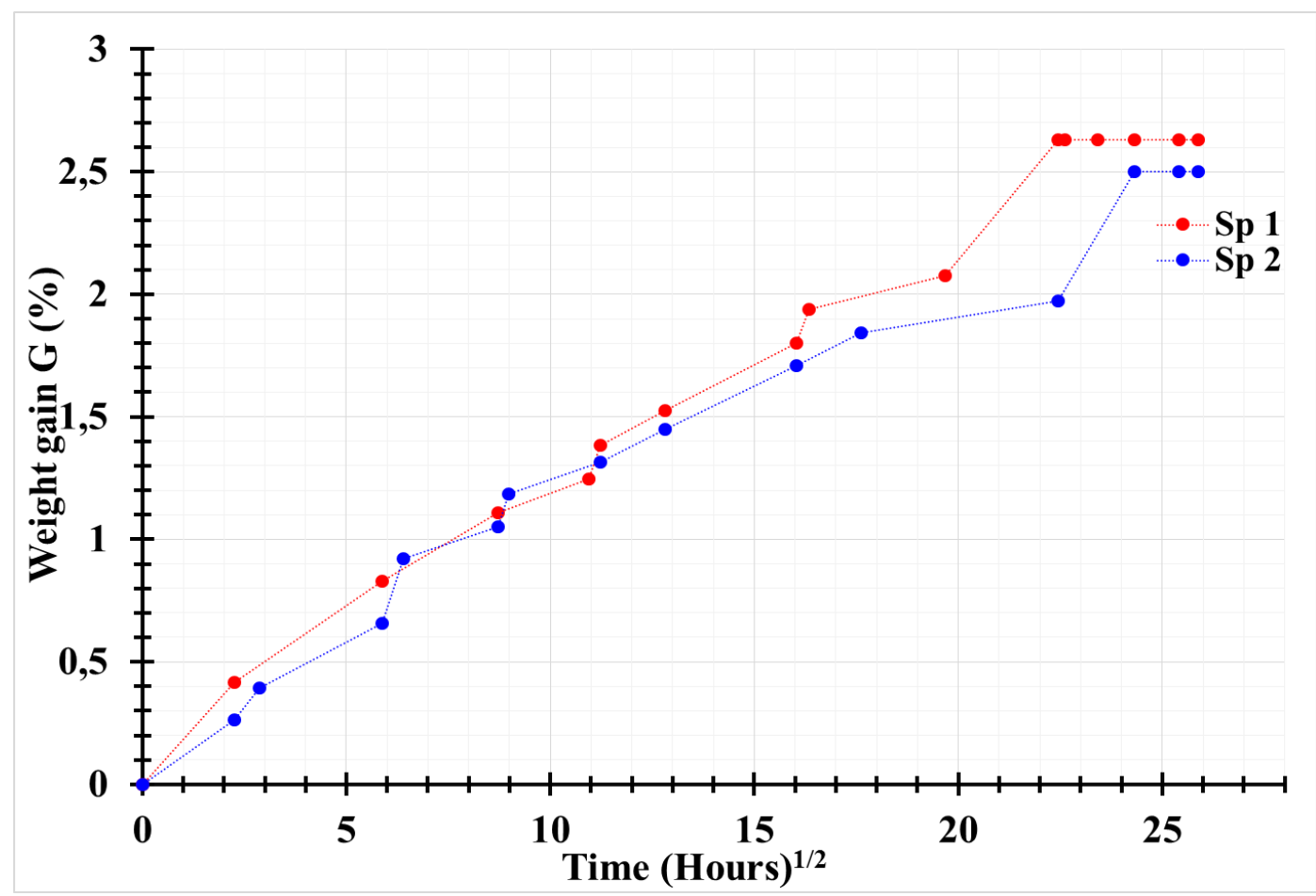

Fig.24. : Water absorption curves of hydrolysis at $\mathrm{T}_{\mathrm{amb}}$

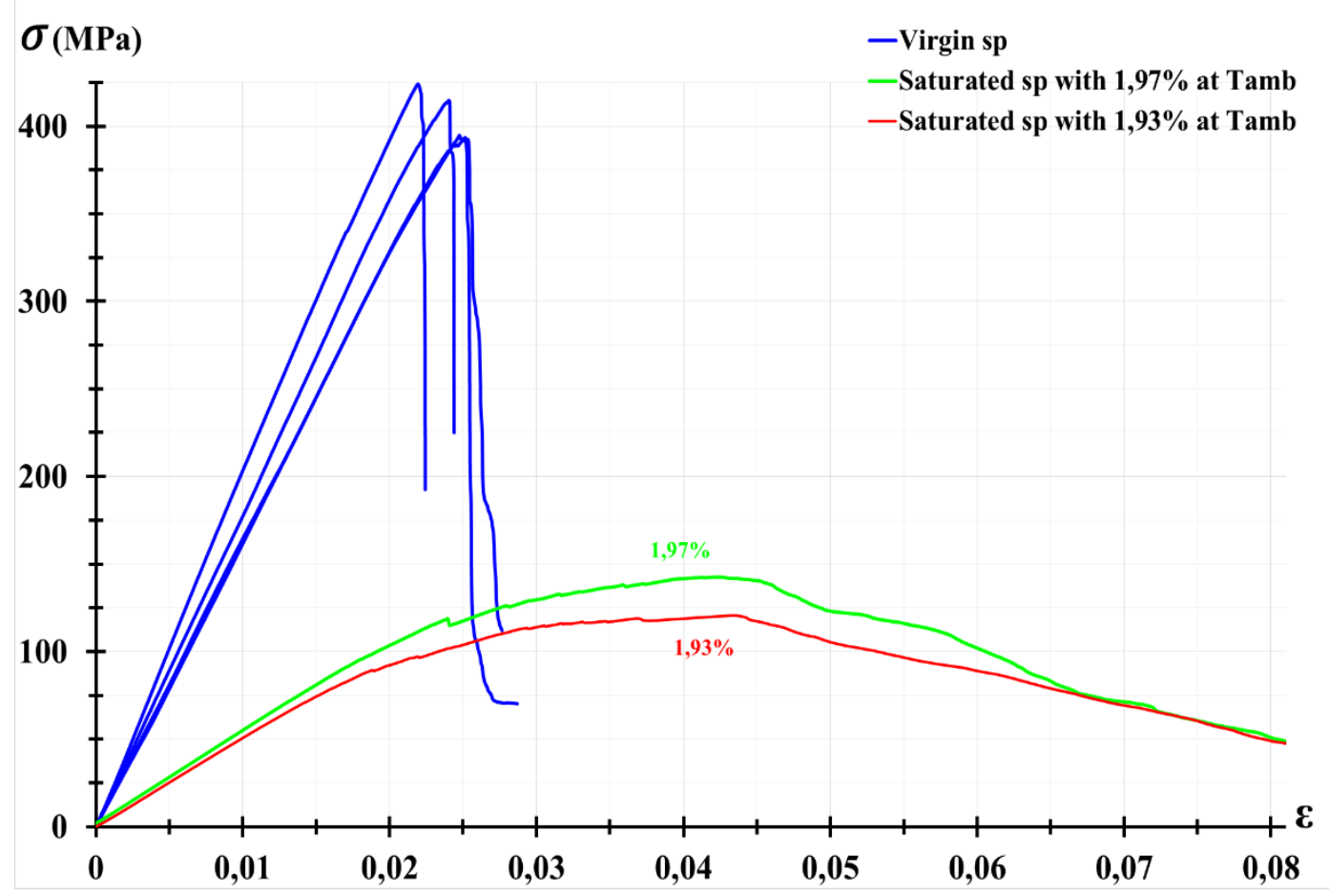

Fig.25. : Result of flexural bending of LU1 before and after hydrolysis at $\mathrm{T}_{\mathrm{amb}}$

To more understand the effect of the hydrolysis temperature on the mechanical behavior of composite, Fig. 26 indicates the water absorption curves of hydrolysis at $80^{\circ} \mathrm{C}$ of LU1 composite samples. After this hydrolysis and a 3 days drying at room temperature, tests of flexural bending are carried out at $\mathrm{T}_{\mathrm{amb}}$. Fig. 27 shows the results of these flexural bending tests of composite samples after hydrolysis at $80^{\circ} \mathrm{C}$ (black curves). 
Their flexural resistance $(\sim 130 \mathrm{MPa})$, is almost equal to the samples which underwent hydrolysis at $\mathrm{T}_{\mathrm{amb}}$. Their Young modulus $\mathrm{E}$ is higher but their deformation rate is smaller by $30 \%$ than the saturated samples at $\mathrm{T}_{\mathrm{amb}}$. Thus, a hydrolysis temperature of $80^{\circ} \mathrm{C}$ does not significantly degrade the mechanical properties of glass fibers but it damages the resin-glass fibers interface, because we have obtained almost the same mechanical properties as $T_{a m b}$ hydrolysis.

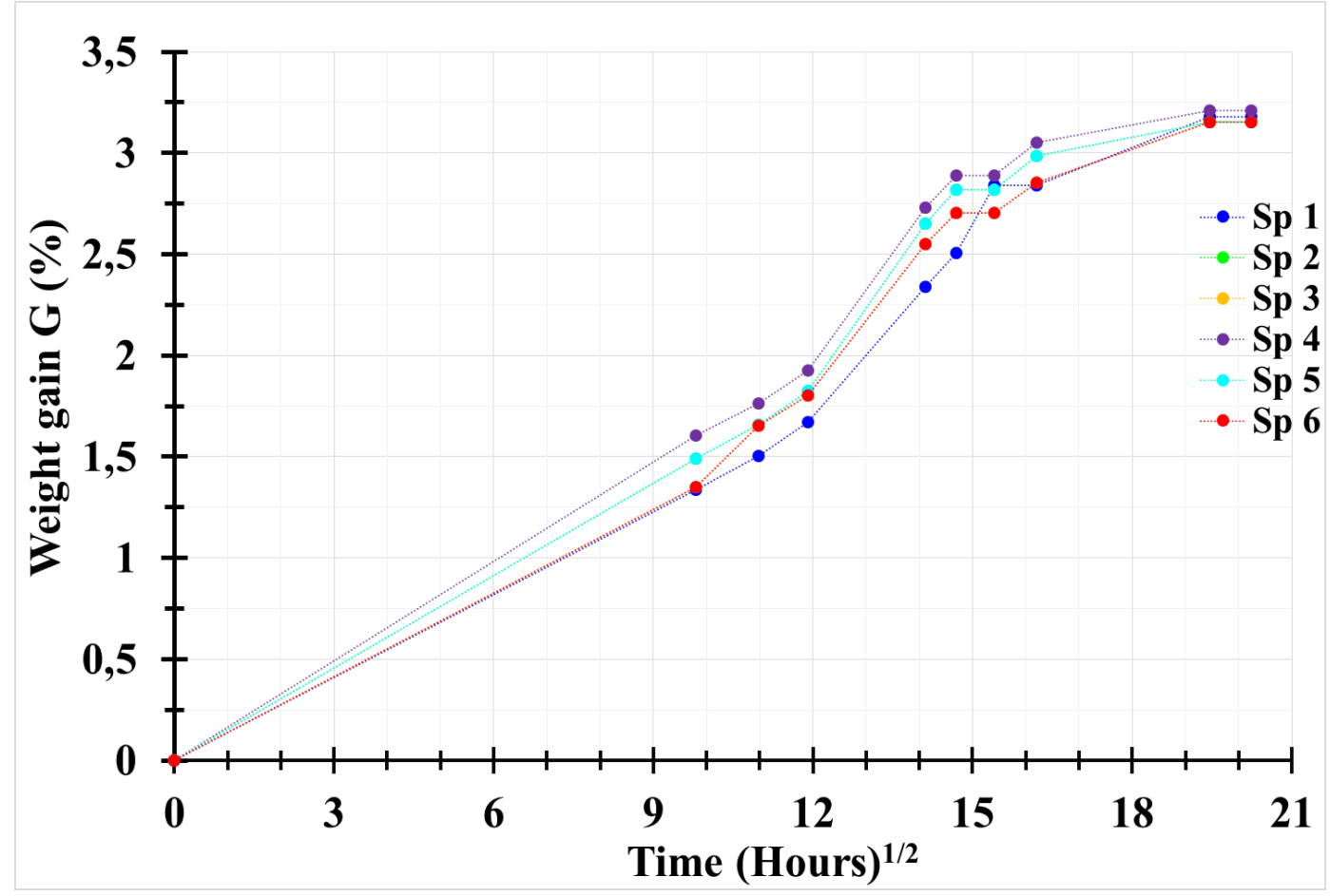

Fig.26. : Water absorption curves of hydrolysis at $80^{\circ} \mathrm{C}$

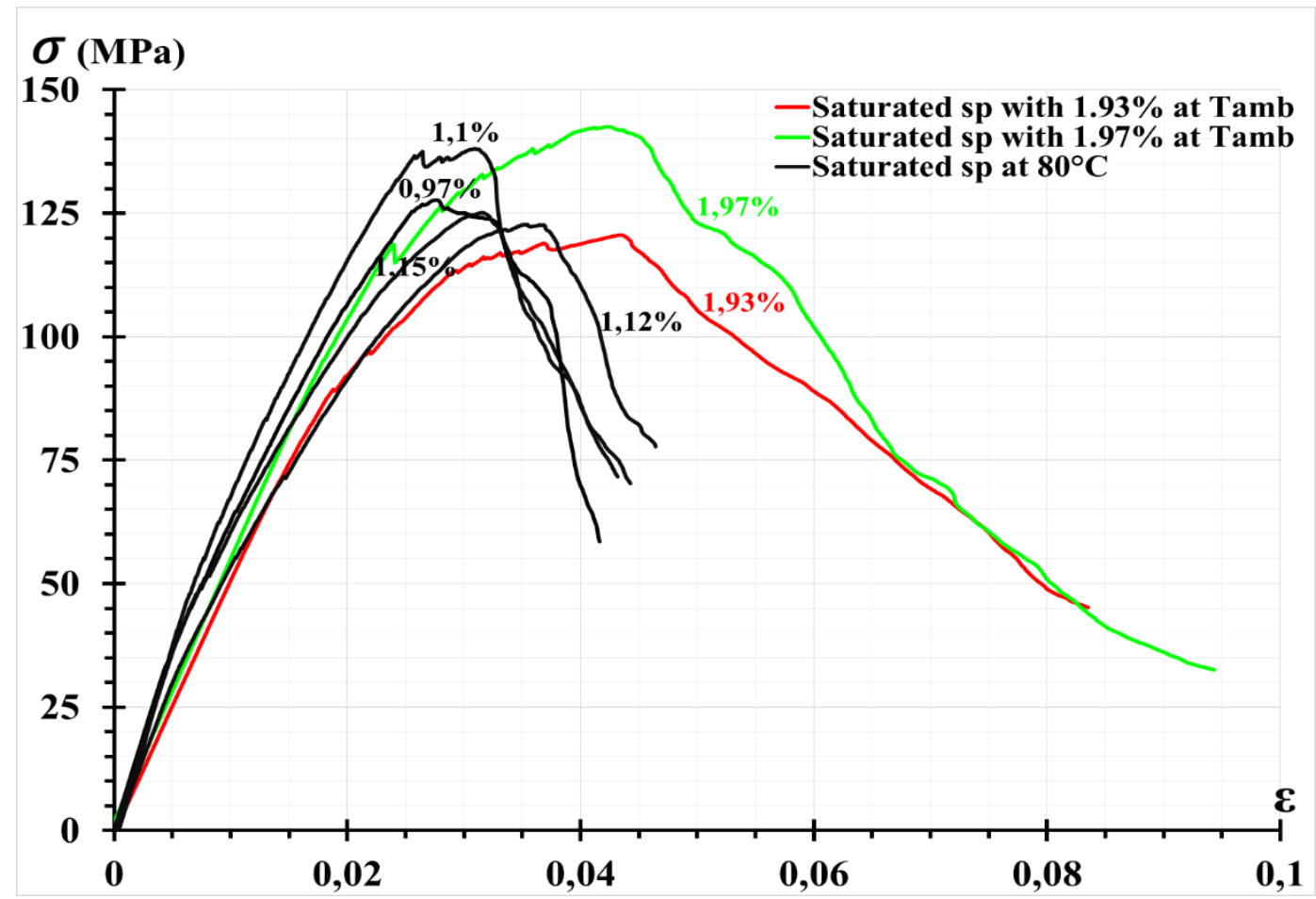

Fig.27. : Result of flexural bending of LU1 after hydrolysis at $80^{\circ} \mathrm{C}$ and at $\mathrm{T}_{\mathrm{amb}}$

To check if the period of drying of these samples after hydrolysis has a significant effect on their compliance, a lot of 3 other samples, which underwent the same hydrolysis protocol, are dried for 7 days at $\mathrm{T}_{\text {amb. Fig. } 28}$ shows the results of the comparison of flexural bending tests of LU1 composite samples after hydrolysis at $80^{\circ} \mathrm{C}$ 
and 3 days drying (black curves) and after 8 days drying at room temperature (orange curves). We notice that the longest drying presents a flexural resistance, $\mathrm{R}_{\mathrm{f}} \sim 148 \mathrm{MPa}$, which is slightly higher than the shortest one. Their young modulus $\mathrm{E}$ is also slightly higher. But all these samples (orange and black) have close failure deformation.

So, we conclude that the drying period has a slight influence on the flexural resistance. So that, a sample composite with a higher water content present a lower flexural resistance. But this period has a significant effect on the breaking strength and on the plastic zone.

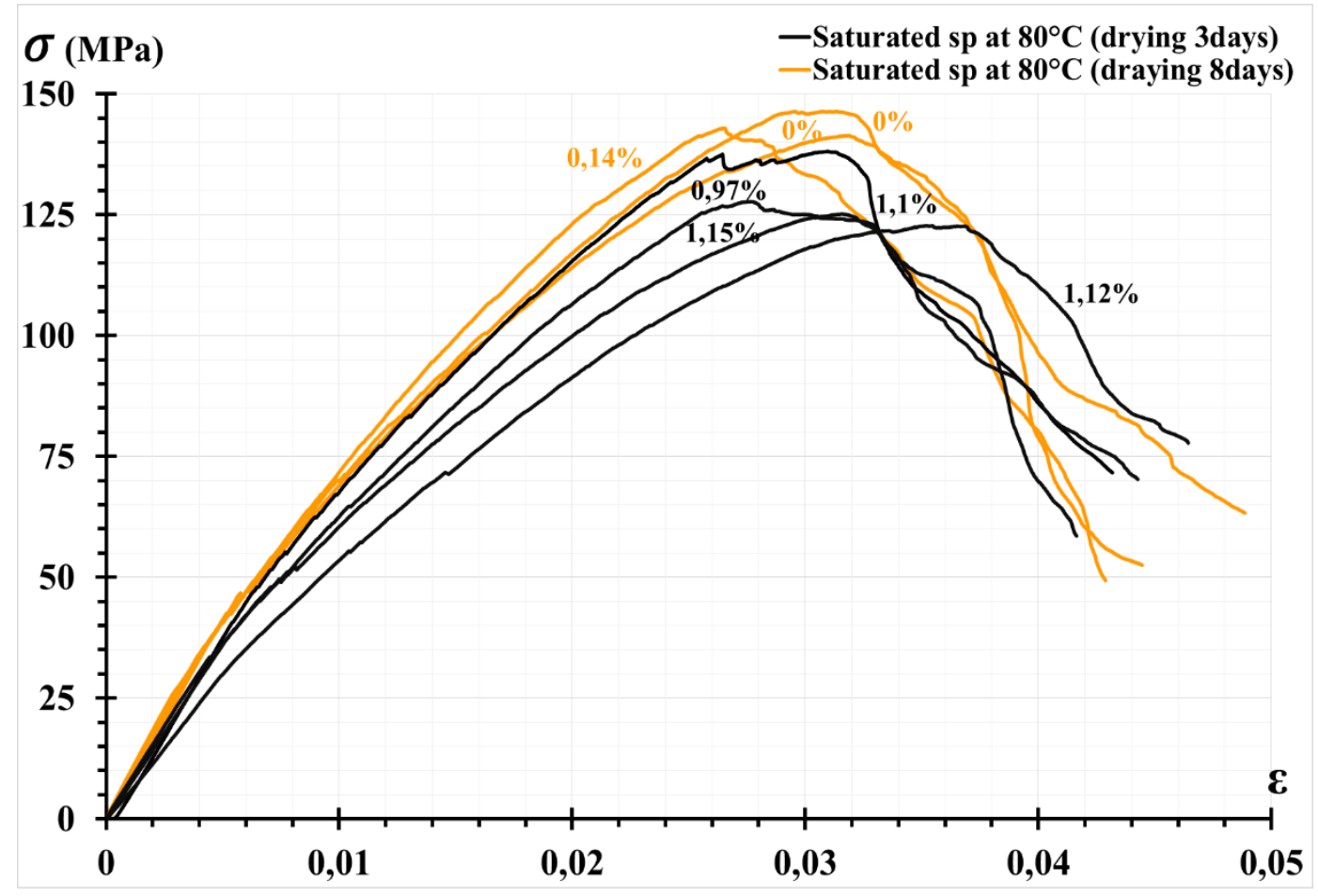

Fig. 28 : Result of flexural bending of LU1 after hydrolysis at $80^{\circ} \mathrm{C}$

To study the influence of hydrolysis on the composite behavior at temperature close to its $\mathrm{T}_{\mathrm{g}} \sim 100^{\circ} \mathrm{C}$, an hydrolysis test of composite is carried out, at $90^{\circ} \mathrm{C}$.

Fig.29 indicates the water absorption curves of samples while hydrolyzing at $90^{\circ} \mathrm{C}$. The purple curves of Fig. 30 are the flexural bending curves of LU1 samples after hydrolysis at $90^{\circ} \mathrm{C}$ and 3 hours and 30 minutes of drying at $90^{\circ} \mathrm{C}$. A comparison of these samples with the samples hydrolyzed at $80^{\circ} \mathrm{C}$ and dried for 8 days, is chosen because all these samples have close water saturation rates.

We note that for both protocols, the composite samples have the same Young modulus E. But the samples hydrolyzed at $90^{\circ} \mathrm{C}$ and allowed to dry 3.3 hours at $90^{\circ} \mathrm{C}$, have a gain of $7 \%$ in the flexural resistance and a gain of $24 \%$ in failure deformation compared to the samples hydrolyzed at $80^{\circ} \mathrm{C}$. Indeed, the samples after this hydrolysis protocol have a loss of $5 \%$ in failure deformation and a loss of $2 \%$ in their flexural resistance, compared to the hydrolyzed composite samples at $\mathrm{T}_{\mathrm{amb}}$ (Fig. 31). The properties are slightly better for drier samples but far from the virgin ones. This drop of composite resistance could be a result of the hydrolysis of the ester bounds of the matrix or of the de-bounding of the matrix - fibers interface or of some degradation of glass fibers. It was shown that the hydrolysis at elevated temperature damages the glass fibers[4, 7].

So, the ageing of composite using the hydrolysis technique at $\mathrm{T}_{\mathrm{amb}}$ and at temperatures close to its $\mathrm{T}_{\mathrm{g}}$, degrades its mechanical properties but it gives to it more compliance. We have obtained a gain of 200\% in its break deformation rate which can facilitate its forming compared to its virgin state.

If we consider the used virgin composite as a production waste type and the hydrolyzed composites as end of life wastes of composites, we remark that the end life wastes have a gain of about $200 \%$ in its break deformation rate compared to the production wastes. That make them more favorable to recycling, considering their high deformation rate compared to new composites or to composite production wastes. 


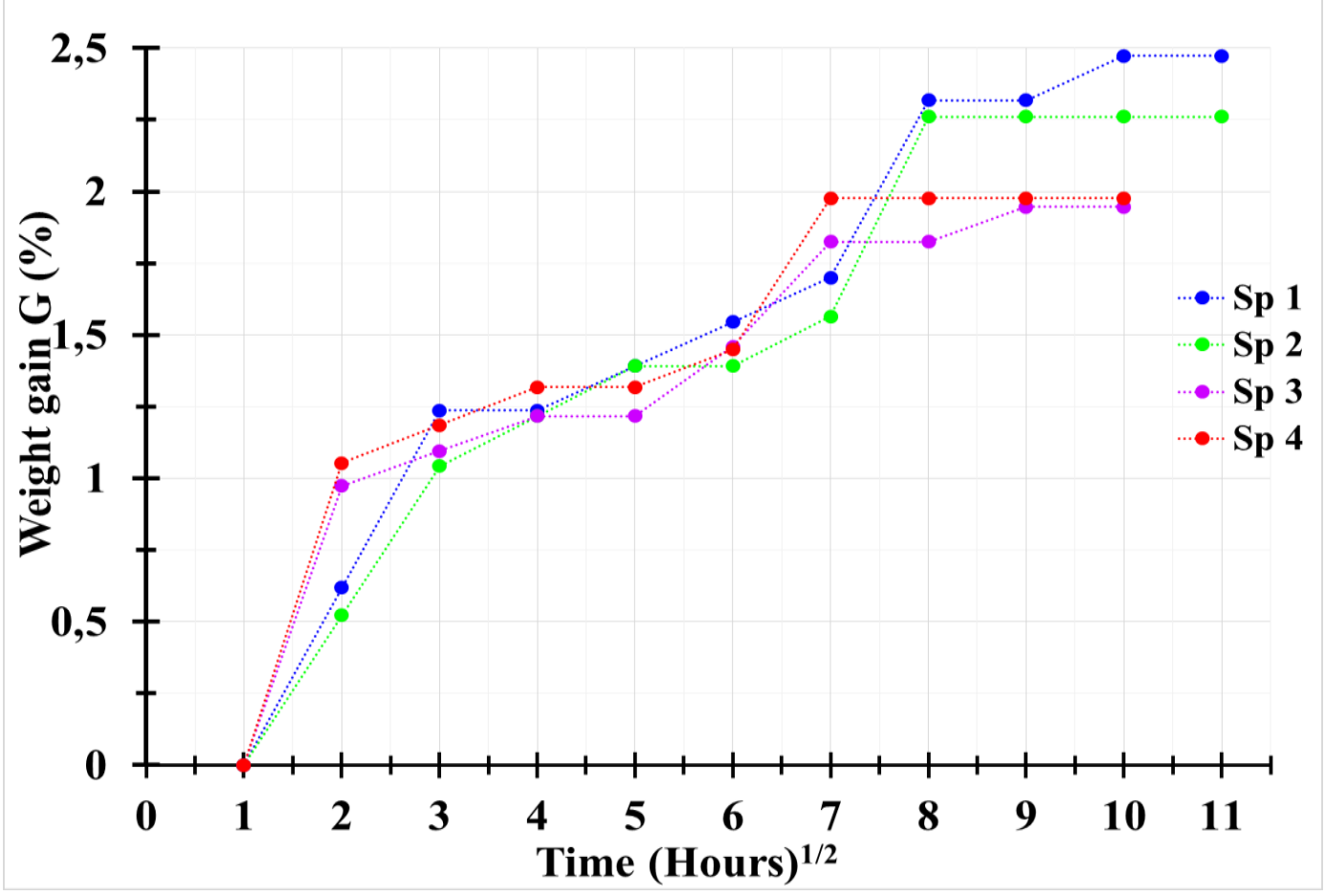

Fig. 29 : Water absorption curves of hydrolysis at $90^{\circ} \mathrm{C}$

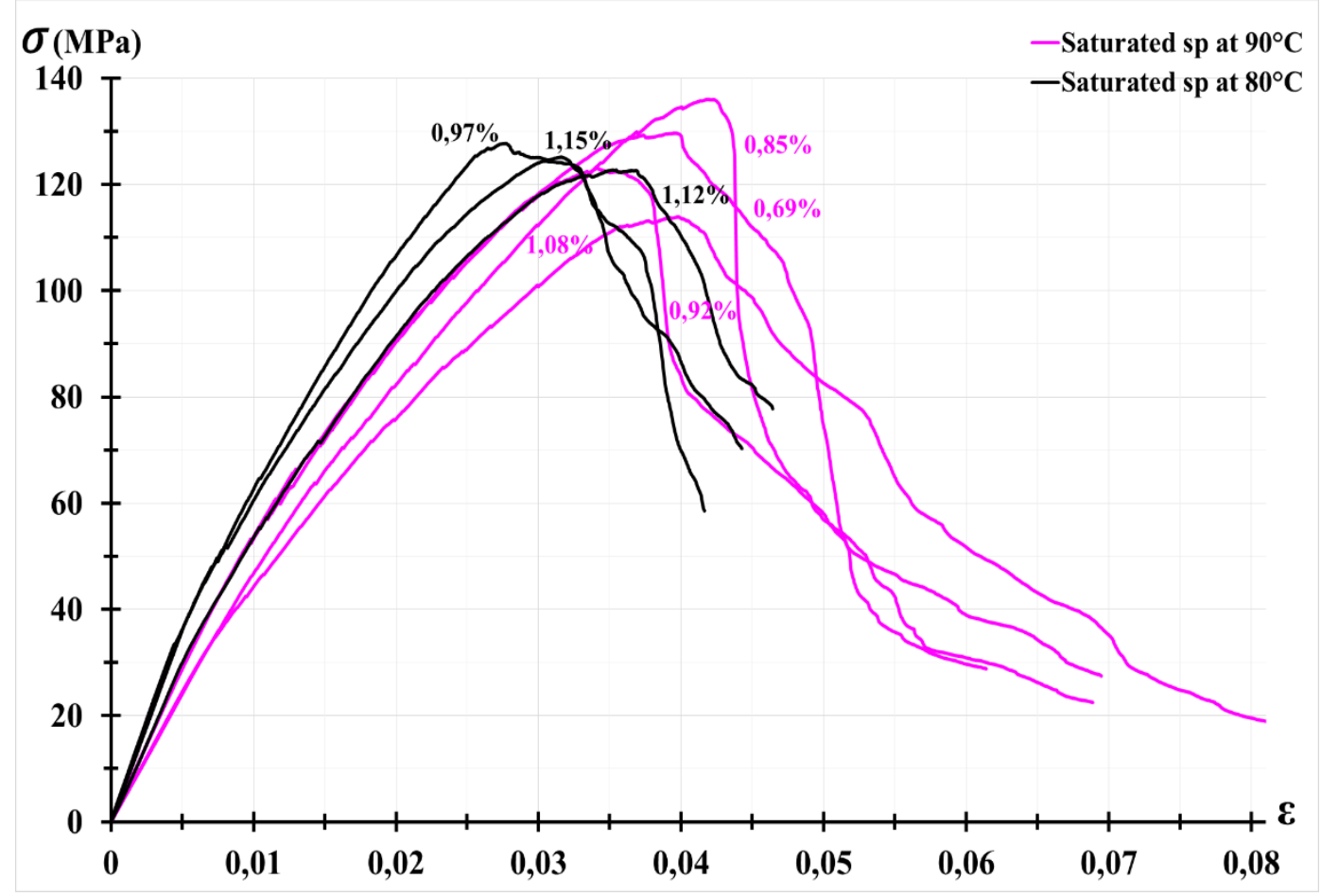

Fig. 30 : Result of flexural bending of LU1 after hydrolysis at $90^{\circ} \mathrm{C}$ and $80^{\circ} \mathrm{C}$ 


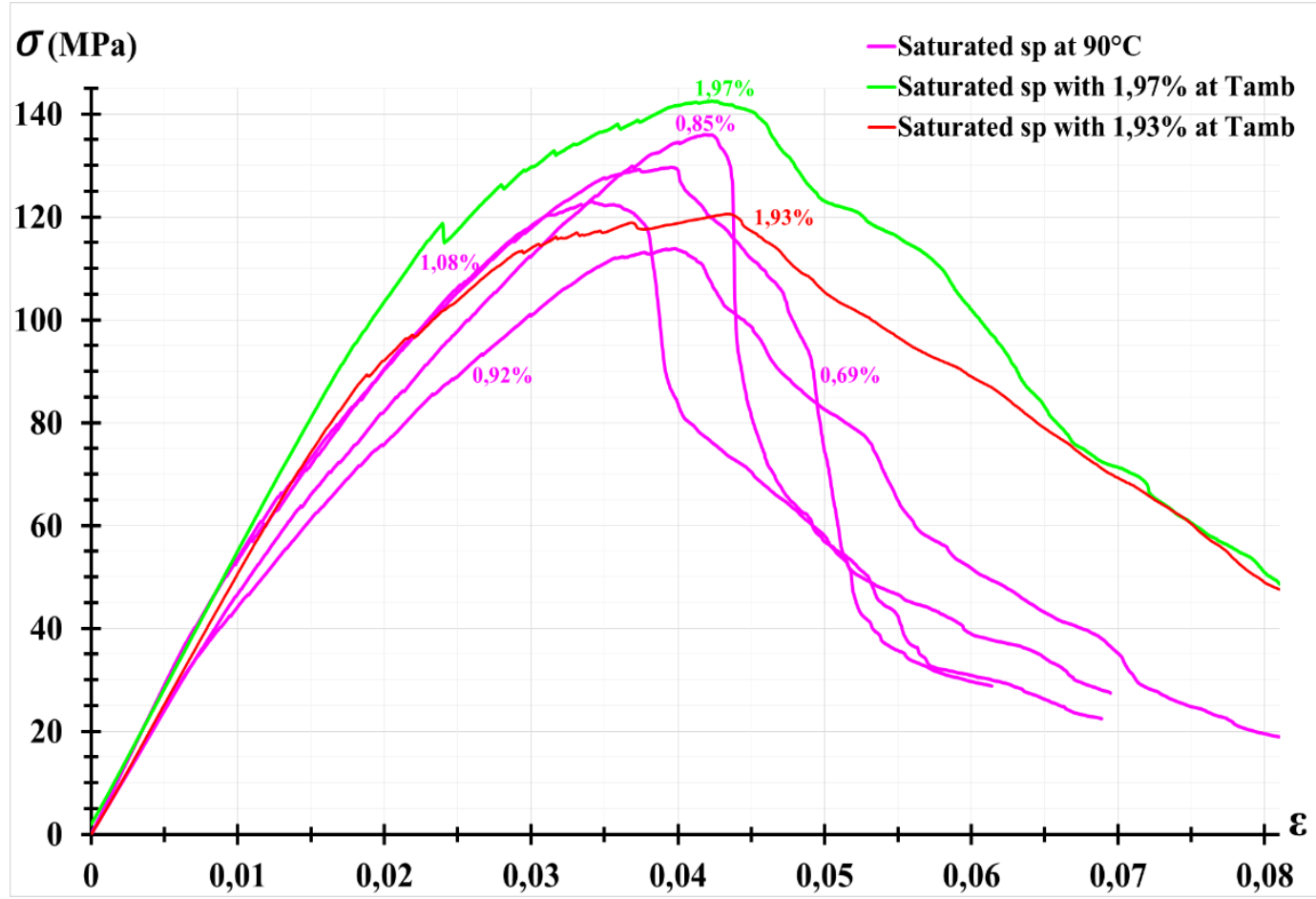

Fig. 31 : Result of flexural bending of LU1 after hydrolysis at $90^{\circ} \mathrm{C}$ and $\mathrm{T}_{\mathrm{amb}}$

\section{Conclusion}

The aim of this study is to define and determine the mechanical properties of polyester-glass fibers composites under an already developed recycling process (thermomechanical process) and to use these measurements to forecast and design the future processes. This process works at relatively high temperatures (around $150^{\circ} \mathrm{C}$ ) and with both production and of end-life-wastes. So, the present work determines experimentally the thermomechanical behavior of models unsaturated polyester resin reinforced with two glass fibers morphologies (chopped and woven roving). The DSC tests revealed the thermal properties of various used composites LU1, LU2 and IN such as their glass transition temperatures which are around $123^{\circ} \mathrm{C}$ for LU1 and LU2 and around 127 for IN, and their specific heat capacities $\mathrm{C}_{\mathrm{p}}$. Also, based on this test, it has found that we can obtain a quasi-cross-linked resin (composite), if we put it at $90^{\circ} \mathrm{C}$ during two hours

The results of static tensile and bending flexural show that composite reinforced woven roving glass LU2 has revealed a better resistance than the composite reinforced chopped and woven roving LU1 at different temperatures from ambient temperature to $150^{\circ} \mathrm{C}$. From $120^{\circ} \mathrm{C}$, the behaviors of all composites used in this study, are changed from rigid to soft viscoelastic or visco-elastoplastic. But their mechanical properties seems always too high for the planned recycling path.

The results of dynamic 3 points bending flexural tests demonstrate the decrease of Young modulus with the increase of temperature which can be contributed to the drop of stiffness composite. Also, it was found that this modulus decreases with the rise of strain level, showing a Payne effect. Indeed, in these dynamic tests, the hysteresis phenomena are noticed at all temperatures.

All the previous dynamic and static tests proved that composite made by infusion is more resistant than the ones made by the hand-lay-up, even when the temperature increases to $150^{\circ} \mathrm{C}$. In addition, composite infusion has the most stable behavior at dynamic test.

For the ageing study of our composite formed by hand lay-up, this material shows many phenomena in the two used techniques: cyclic tensile test and hydrolysis at different temperatures. The results of cyclic tensile tests show hysteresis phenomena and Mullins effect at $150^{\circ} \mathrm{C}$. At this temperature, this composite is in its rubbery state.

The water ageing tests show that the composite, hydrolyzed at $\mathrm{T}_{\mathrm{amb}}$, lost about $66 \%$ of its flexural resistance but gains $200 \%$ in its failure deformation. For hydrolysis at temperatures close to its $\mathrm{T}_{\mathrm{g}}$ (glass transition temperature $\sim 100^{\circ} \mathrm{C}$ ), the composite has a $170 \%$ gain of its failure deformation but a $60 \%$ loss of flexural resistance. So, the hydrolysis at $\mathrm{T}_{\mathrm{amb}}$ or the accelerated one at temperatures close to $\mathrm{T}_{\mathrm{g}}$ give a significant compliance to the materials 
but degrade its resistance. Thus, water absorption is thought to degrade the matrix, the fiber-matrix interfaces and to damage the glass fibers properties, which were not recovered by a further drying.

If the virgin composites (before hydrolysis) are considered as production wastes and the hydrolyzed composites as a type of end of life wastes, an important gain of composite failure deformation is obtained for the hydrolyzed composites compared to the virgin ones. Thus, it means that end of life wastes should be more easily recycled than production wastes, because the degree of total deformation of end life wastes (hydrolyzed composites) is higher than the production wastes (virgin composites).

\section{Funding}

This study was funded by FEDER - Region Pays de la Loire in the framework of CIPTAP R\&D project.

\section{Conflict of interest}

The authors declare that they have no conflict of interest.

\section{References}

[1] Cetim C, (2015) Composites et recyclage: état de l'art et perspectives. Journées promotion Procédés Produits. https://www.progepi.fr/wp-content/uploads/2017/04/f.-ruch-cetim-cermat.pdf

[2] Vannessa G, Vannessa Goodship (2010) Management, recycling and reuse of waste composites. Woodhead Publishing, New York Washington.

[3] Website: 'http://www.statistiques.developpement-durable.gouv.fr/indicateurs-indices/f/1929/0/tauxrecyclage-dechets-france.html'.

[4] Oliveux G, Dandy L-O, Leeke A-G, (2015) A Current status of recycling of fibre reinforced polymers: Review of technologies, reuse and resulting properties. Progress in Materials Science (72): 61-99,

[5] Li D, Kaewunruen S, (2019) Mechanical properties of concrete with recycled composite and plastic aggregates. International Journal of Geomate (17) 60: 231-238, Japan.

[6] Li W, Xiao J, Shi C, Poon C-S, (2015) Structural behaviour of composite members with recycled aggregate Concrete - an Overview. Advances in Structural Engineering 6 (18).

[7] Oliveux G, Bailleul J-L, L-G-L-Salle E, Lefèvrea N, Biotteau G, (2012) Recycling of glass fibre reinforced composites using subcritical hydrolysis: Reaction mechanisms and kinetics, influence of the chemical structure of the resin. Polymer Degradation and Stability: 1-16.

[8] 'AB Val composites: 3,8 millions d'euros pour un recyclage inédit | L'Éclaireur de Châteaubriant'. https://actu.fr/economie/ab-val-composites-38-millions-deuros-pour-un-recyclage-inedit_8685701.html

[9] Wazery M-S, EL-Elamy M-I, Zoalfakar S-H, (2017) Mechanical Properties of Glass Fiber Reinforced Polyester Composites. International Journal of Applied Science and Engineering 3 (14): 121-131.

[10] Abdullah E-T, (2013) A Study of Bending Properties of Unsaturated Polyester/Glass Fiber Reinforced Composites. Journal of Al-Nahrain University Science 3 (16): 129-132.

[11] Davallo M, Pasdar H, (2009) Comparison of Mechanical Properties of Glass-Polyester Composites Formed by Resin Transfer Moulding and Hand Lay-Up Technique. International Journal of ChemTech Research $1(3)$

[12] Laoubi K, Hamadi Z, Benyahia A, Serier A, Azari Z, (2014) Thermal behavior of E-glass fiber-reinforced unsaturated polyester composites. Composites Part B: Engineering (56): 520-526.

[13] Dhakal H-N, Zhang Z-Y, Richardson M-O-W, (2006) Effect of water absorption on the mechanical properties of hemp fibre reinforced unsaturated polyester composites. Composites Science and Technology.

[14] Perrot Y, (2006) Influence des propriétés de la matrice sur le comportement mécanique de matériaux 
composites verre/polyester utilisés en construction navale de plaisance - Cas des résines polyester limitant les émissions de styrène. Université de Bretagne Sud.

[15] Bouazza H, (2011) Recyclage des matériaux composites thermodurcissables à usage naval. Université des sciences et de la technologie d'Oran.

[16] Giljean S, Ibrahim A, L'Hostis G., (2017) Influence du mode de polymérisation de composites verre/polyester sur leur comportement mécanique. 23ème Congrès Français de Mécanique: 6.

[17] Liang S, (2012) Etude de comportement en fatigue des composites renforcés par fibres végétales. Prise en compte de la variabilité des propriétés. Université de Bourgogne.

[18] Sanga R-P-L, Ntamack G-E, Mansouri K, Beda T, D’Ouazzane S-C, (2013) Evaluation des Caractéristiques Mécaniques des Matériaux Composites en Fonction de la Température, Application au Composite Epoxyde + Verre. Journal of Materials and Environmental Science 4 (6): 103-104.

[19] Hentschke R, (2017) The Payne effect revisited. eXPRESS Polymer Letters 4 (11): 278-292.

[20] Hassine M.B, (2013) Modélisation du vieillissement thermique et mécanique d'une protection externe en EPDM de jonctions rétractables à froid. Ecole doctorale de Sciences des Métiers de l'Ingénieur, Paris Tech.

[21] Huger M, (2008) Elasticité à haute température de matériaux céramiques : effet des hétérogénéités. Ecole Nationale Supérieure de Céramique Industrielle.

[22] Luo R-K, (2015) Mullins damage effect on rubber products with residual strain. International Journal of Damage Mechanics 2 (24): 153-167.

[23] Shirinbayan M, (2017) Étude du comportement mécanique et de l'endommagement de divers matériaux composites SMC soumis à des chargements de type dynamique, fatigue et dynamique post-fatigue. École Nationale Supérieure d'Arts et Métiers.

[24] Chagnon G, Marckmann G, Verron E, Gornet L, O-Kuczynski E, Charrier P, (2016) A new modelling of the Mullins' effect and viscoelasticity of elastomers based on physical approach. Ecole Centrale de Nantes, Groupe Trelleborg: Société Modyn Nantes.

[25] Bhave T, Tehrani M, Ali M., Sarvestani A, (2018) Hysteresis friction and nonlinear viscoelasticity of rubber composites. Composites Communications (9): 92-97. 


\section{Cover letter}

February, 2020

\section{Dear Editor,}

I am submitting the manuscript entitled "Thermo-mechanical characterization of unsaturated polyester/glass fiber composites for recycling" for consideration for publication in the Journal of Materials International Journal of Material Forming.

We warrant that the article has not received prior publication and is not under consideration for publication elsewhere. This research has not been submitted for publication nor has it been published in whole or in part elsewhere.

Best wishes,

\section{Arbia Nouigues}

Icam de Nantes, 35 avenue du champ de Manœuvres, 44470 Carquefou, Nantes

LTeN, UMR CNRS 6607, Rue Christian Pauc, 44306, Nantes

E-mail : Arbia.nouigues@icam.fr

Arbia.nouigues@etu.univ-nantes@fr 